Primljen / Received: 21.1.2020 Ispravljen / Corrected: 20.5.2021

Prihvaćen / Accepted: 15.10.2021.

Dostupno online / Available online: 10.11.2021.

\section{Numerical analysis of RC columns under cyclic uniaxial and biaxial lateral load}

Authors:

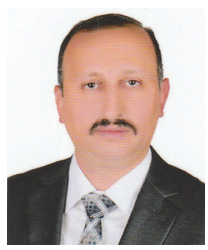

Assist.Prof. Ibrahim S.I. Harba, PhD. CE

Al-Nahrain University, Baghdad, Iraq

College of Engineering

Department of Civil Engineering

lbrahim.S.Ibrahim@nahrainuniv.edu.iq

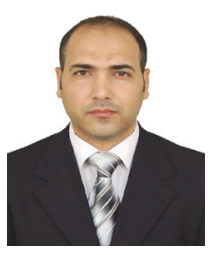

Assist.Prof. Abdulkhalik J. Abdulridha, PhD. CE

Al-Nahrain University, Baghdad, Iraq

College of Engineering

Department of Civil Engineering

Abdulkhalik.J.AbdulRidha@nahrainuniv.edu.iq Corresponding author
Research Paper

Ibrahim S.I. Harba, Abdulkhalik J. Abdulridha

\section{Numerical analysis of RC columns under cyclic uniaxial and biaxial lateral load}

A numerical finite element study is conducted in this paper to examine structural behaviour of high strength RC columns exposed to biaxial and uniaxial lateral displacement histories with constant axial load. The numerical analysis of 24 models was made using ABAQUS / CAE. The comparison between numerical analysis and experimental results shows good agreement through validations. The considered parametric study involves determination of the longitudinal reinforcement ratio, total cross-sectional area of confinement steel (Ash), and uniaxial and biaxial cyclic shear load. Numerical analysis results show that an increase of longitudinal reinforcement for a uniaxial and biaxial lateral historic load will significantly increase maximum and ultimate load of columns, corresponding deflections, number of cycles at maximum and ultimate loads, and initial stiffness Ki, while the effect of transverse reinforcement is less pronounced. The columns load and deformation capacity decreases significantly with application of biaxial cyclic shear load, compared with uniaxial load. Also, this effect reduces with an increase in longitudinal reinforcement ratio $\left(\% \rho_{1}\right)$ and Ash.

Key words:

columns, high-strength concrete, numerical analysis, numerical simulation, transverse historic load, uniaxial and biaxial load

Prethodno priopćenje

Ibrahim S.I. Harba, Abdulkhalik J. Abdulridha

\section{Numerička analiza cikličnog jednoosnog i dvoosnog bočnog opterećenja AB stupova}

U ovom se radu prikazuje numerička analiza konačnih elemenata koja je provedena kako bi se ispitalo ponašanje AB stupova visoke čvrstoće za slučaj dvoosnog i jednoosnog bočnog pomaka pri konstantnom uzdužnom opterećenju. Numerička analiza provedena je na 24 modela pomoću programa ABAQUS / CAE. Validacijom je utvrđena dobra podudarnost numeričkih i eksperimentalnih rezultata. U okviru parametarske studije određen je koeficijent armiranja uzdužnom armaturom, ukupna ploština spona za ovijanje (Ash) te jednoosno i dvoosno ciklično posmično opterećenje. Rezultati numeričke analize pokazuju da povećanje količine uzdužne armature u slučaju jednoosnog i dvoosnog bočnog opterećenja dovodi do znatnog povećanja maksimalnog i graničnog opterećenja stupova, progiba, broja ciklusa maksimalnog i graničnog opterećenja, te početne krutosti Ki, dok je utjecaj poprečne armature manje izražen. Otpornost stupova na opterećenja i deformacije znatno se smanjuje pri nanošenju dvoosnog cikličnog posmičnog opterećenja, u odnosu na jednoosno opterećenje. Isto tako, taj utjecaj se smanjuje s povećanjem udjela uzdužne armature $\left(\% \rho_{1}\right)$ i vrijednosti Ash.

Ključne riječi:

stupovi, beton visoke čvrstoće, numerička analiza, numerička simulacija, prethodno poprečno opterećenje, jednoosno i dvoosno opterećenje 


\section{Introduction}

The current investigation, conducted within the topic on seismic behaviour of RC structures widely studied over the past several decades, focuses on the performance of RC columns subjected to axial and biaxial horizontal load [1, 2]. Structural response registered during earthquakes reveals that high shear stresses, decrement of concrete material characteristics, and the effect of bidirectional loading, have been a major cause of column failure [3]. It is quite clear that earthquake damage to RC elements is due to multi-axial excitation. As is generally known from interaction diagrams, both ultimate moments and yielding rise with the amount of the axial load until an equilibrium stage is achieved [4,5].

During an earthquake, a change in axial load can change the ultimate displacement capacity, strength, and stiffness, in addition to all hysteretic properties of an RC section. These variations occur due to vertical component of seismic load, or to load exerted at the bottom. At external columns of RC frames, the axial load decreases on the one side and increases on the opposite side, which is caused by overturning moments. In fact, the inelastic response of columns is significantly affected by variations in axial force during the response cycle [6-9].

In general, the cycle of biaxial transversal load increases the degradation in strength and stiffness compared with the uniaxial load. Also, the RC column failure mechanism depends on the path/history of load, and strongly affects ductility and energy dissipation capacity of columns [10-14]. The intensity of axial load has little effect on the specimen load-displacement envelope curve of the second-cycle under variable or constant axial load with unidirectional horizontal load [15].

Several studies have shown that an increase in compressive strength of concrete decreases displacement ductility of columns. On the other hand, the effect of high strength concrete (HSC) on the ductility (drift limit) of columns is still questionable [16].

The effectiveness of confinement and ultimate strain of columns decreases with an increase in the strength of concrete. Specimens with a ratio of $1.1 \%$ of confining reinforcement will give inadequate ductility, while with 3.1 $\%$ transverse reinforcement the specimen's behaviour is satisfactory[17].

The ductility (drift ratio) of HSC columns is highly affected by the capacity of lateral reinforcement and the level of axial compression [18-20]. For specimen drift ratios exceeding $4 \%$ with an axial load of $0.3 \mathrm{fcAg}$, a strong correlation was observed between the drift limit and axial load. Specimens failed in shear at higher axial load. For columns subjected to axial loads exceeding the balanced load, a large amount of transverse reinforcement was required to obtain ductile behaviour [21]. In this paper, the advanced "concrete damage plasticity" (CDP) was used in the numerical analysis of 24 models using ABAQUS software. Numerical model was validated by comparison with experimental tests [21] as a first step before conducting a parametric study to develop a numerically validated model. The following parameters were studied in this paper: longitudinal reinforcement ratio, total cross-sectional area of confinement steel (Ash), and uniaxial and biaxial cyclic displacement shear load, in order to examine structural behaviour of high strength $\mathrm{RC}$ columns.

\section{Research significance}

Several experimental studies have been made on the behaviour of columns under lateral historic load. However, a limited number of studies focus on the effect of longitudinal and lateral reinforcement with uniaxial and biaxial lateral displacement histories on the displacement capacity of HSC columns subjected to constant axial load.

\section{Experimental results considered in the research}

The experimental result used for validating accuracy of the present numerical analysis was presented by Matamoros and Sozen [21]. Eight specimens of RC columns with normal and high strength concrete were tested. This experimental program involved testing of columns subjected to shear reversals, and the main variables were axial load, concrete strength, and loading history. The dimensions and specimen reinforcement detailing are shown in Figure 1. Mechanical properties of concrete and steel reinforcement are listed in Table 1 and Table 2. In addition, the displacement history for each direction was increased by $6.5(\mathrm{~mm})$ for each subsequent two cycles, as shown in Figure 2. The considered specimen C70-20 was used in the current study as control specimen for validation. The numerical analysis was first validated with experimental results using ABAQUS[22] and then the parametric study was developed.

Table 1. Mechanical properties of concrete

\begin{tabular}{|c|c|c|c|c|c|c|}
\hline Specimen & $\begin{array}{c}\text { Axial load } \\
{[\mathrm{kN}]}\end{array}$ & $\begin{array}{c}\text { Axial stress } \\
{[\mathrm{MPa}]}\end{array}$ & $\begin{array}{c}\text { Target compressive } \\
\text { strength }[\mathrm{MPa}]\end{array}$ & $\begin{array}{c}\text { Mean compressive } \\
\text { strength [MPa] }\end{array}$ & $\begin{array}{c}\text { Tensile strength } \\
{[\mathrm{MPa}]}\end{array}$ & $\begin{array}{c}\text { Modulus of elasticity } \\
{[\mathrm{GPa}]}\end{array}$ \\
\hline $\mathrm{C70}-20$ & 568 & 14 & 70 & 66 & 6.4 & 35.9 \\
\hline
\end{tabular}

Table 2. Mechanical properties of steel reinforcement

\begin{tabular}{|c|c|c|c|}
\hline Specimen & Reinforcing bar size & Yield stress [MPa] & Ultimate stress [MPa] \\
\hline C70-20 & 5 & 585 & 745 \\
\hline
\end{tabular}


a)
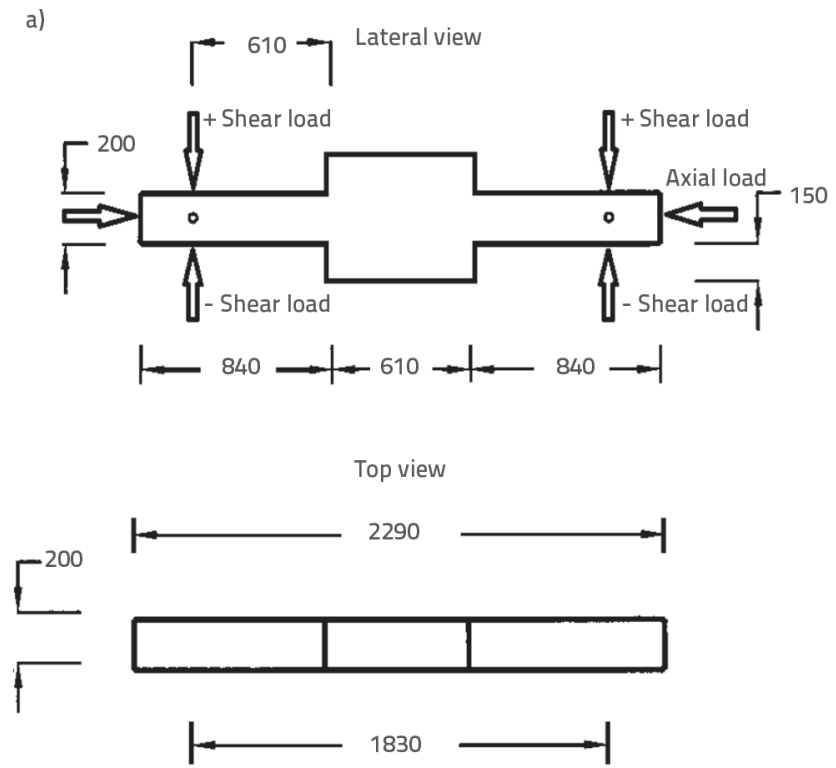

Figure 1. a) Specimen's dimensions; b) reinforcement details [21]

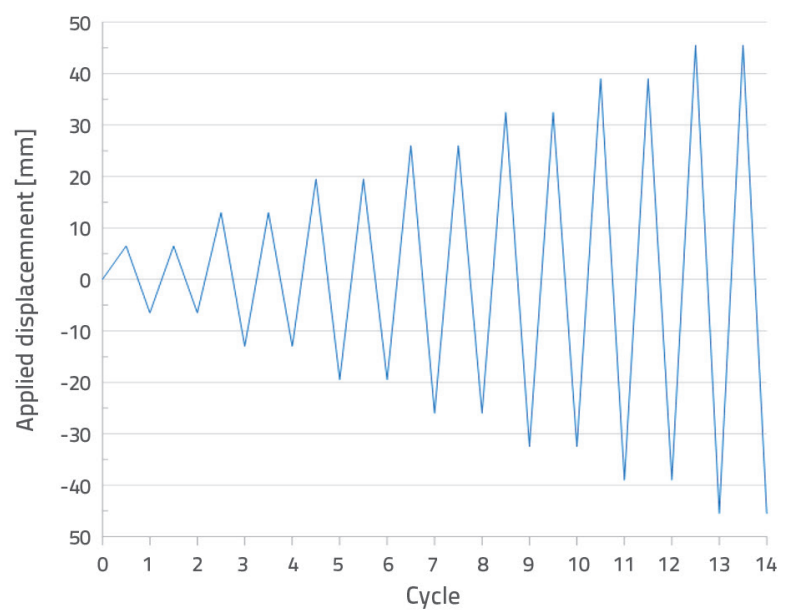

Figure 2. Displacement history for each direction [21]

\section{Numerical modelling}

Numerical modelling was conducted using the ABAQUS software [22]. Nonlinear three-dimensional FE model with material properties was used to build up the RC column. The experimental cyclic behaviour obtained during previous test results [21] was used in the finite element software ABAQUS as input data. The concrete and steel constitutive model, type of element and boundary conditions, as used for numerical modelling, are discussed below.

\subsection{Constitutive model of concrete}

Non-linear constitutive behaviour of concrete was modelled using the "concrete damage plasticity" (CDP) model for defining

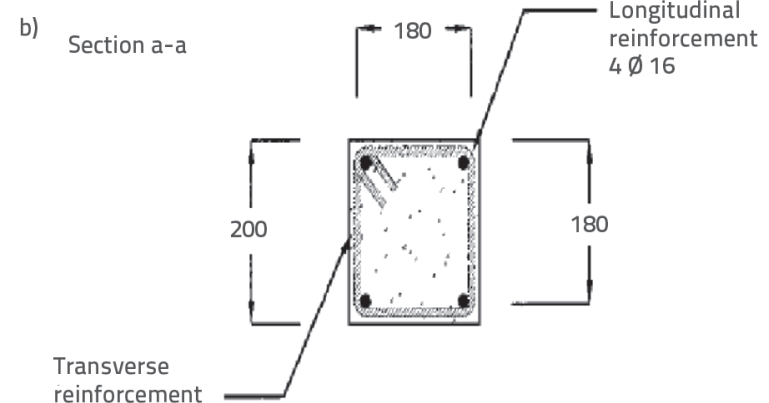

reinforcement $\emptyset 10 / 75$

Distribution of reinforcement

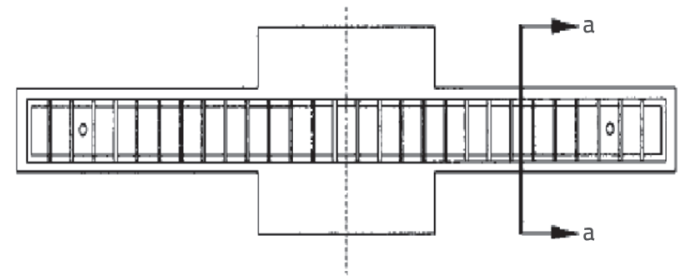

All dimensions in millimeter

cyclic and monotonic behaviour of concrete. The CDP model is defined by five plasticity parameters and concrete stressstrain curves in compression and tension [22, 23] as illustrated in Table 3. In 1985, Carreira and Chu [24] suggested a formula that was adapted to calculate the concrete stress-strain curve in compression. The behaviour of concrete in tension is considered as linear-elastic until the uniaxial tensile stress, at which point the concrete cracks. The behaviour of concrete in tension proposed by Hordijk [25] is adopted in this study.

Table 3. Summary of CDP parameters used

\begin{tabular}{|c|c|c|}
\hline Parameter & Explanation & Value \\
\hline$\psi$ & Dilation angle & 18 \\
\hline$\sigma b 0 / \sigma c 0$ & $\begin{array}{c}\text { Ratio of initial equi-biaxial } \\
\text { compressive yield stress to initial } \\
\text { uniaxial compressive yield stress }\end{array}$ & 0.1 \\
\hline Kc & $\begin{array}{c}\text { The coefficient determining the } \\
\text { shape of the deviatoric cross-section }\end{array}$ & 0.667 \\
\hline$\mu$ & Viscosity parameter & 0.001 \\
\hline
\end{tabular}

\subsection{Constitutive model of steel}

The constitutive model of steel was used to properly simulate behaviour of steel reinforcement under cyclic load reversals, since the yield stress decreases as the number of load cycles increases in the direction of plastic deformation. It was also used to account for the effects of hardening and softening of steel reinforcement. The behaviour is assumed to include 
the bilinear kinematic hardening also considered in relation to Bauschinger effect [26-28]. In this study, the behaviour of longitudinal and transverse steel reinforcement is simulated using the model proposed by Ucak and Tsopelas [29]. To identify a more reasonable behaviour, the input data are measured in relation to the reinforcement tensile test results discussed in the validated experimental test [21].

\subsection{Element, loading and boundary conditions}

In ABAQUS, the concrete was modelled using C3D8R element type and T3D2 element type for modelling the longitudinal and transverse reinforcement. The reinforcement elements were embedded in concrete elements to simulate the bonding correlation between the concrete and reinforcement. A set of steel gaskets was used in the position of applied load and supports to avoid local failure and stress concentration in the RC column loading surface and supports, as shown in Figure 3.

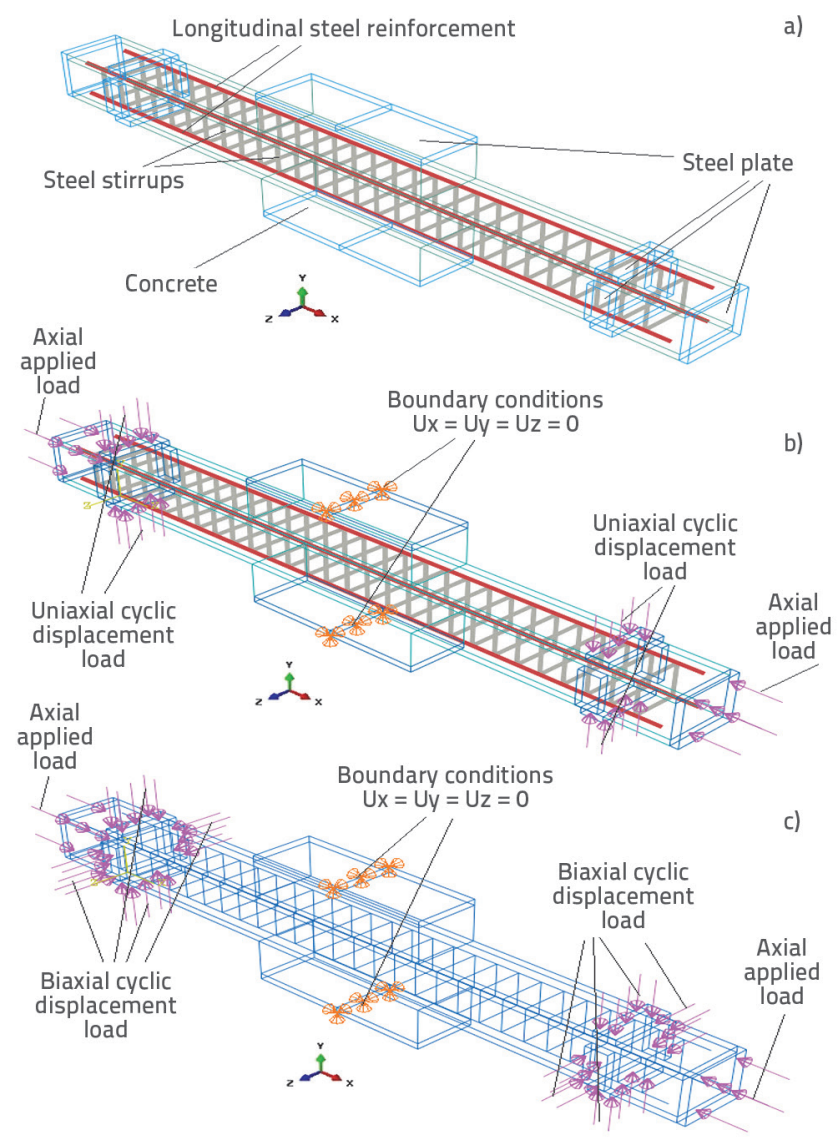

Figure 3. a) Steel reinforcement and steel plates detail; b) uniaxial specimen's load detail; c) biaxial specimen's load detail

During the analysis, various element mesh sizes were used to avoid the convergence problem. Thus, the mesh sizes of $45 \mathrm{~mm}$, $40 \mathrm{~mm}$, and $35 \mathrm{~mm}$ were used in the analysis.

The element size $35 \mathrm{~mm}$ shows accurate FE analysis results when compared with relevant experimental results [21]. To make the simulation in accordance with the real test, all nodes at the top and bottom surfaces of the central stub are totally constrained as fixed boundary conditions. A constant axial load is modelled as compression pressure on steel gasket at the column face in the $x$-direction.

The uniaxial load is simulated by applying the uniaxial cyclic displacement load in the $-Y$ and $+Y$ directions, as shown in Figure $3 b$. The biaxial cyclic displacement load is achieved by applying load in the $-Y$ and $+Z$ directions, and reverse in the $+Y$ and $-Z$ directions, as shown in Figure 3c. The displacement history in both directions is shown in Figure 2.

Meanwhile, the transverse displacement controlled cyclic load is imposed based on the insert amplitude on the free end of the column in Y-direction as shown in Figure 4, which is the same model that has been used in the experimental work [21].

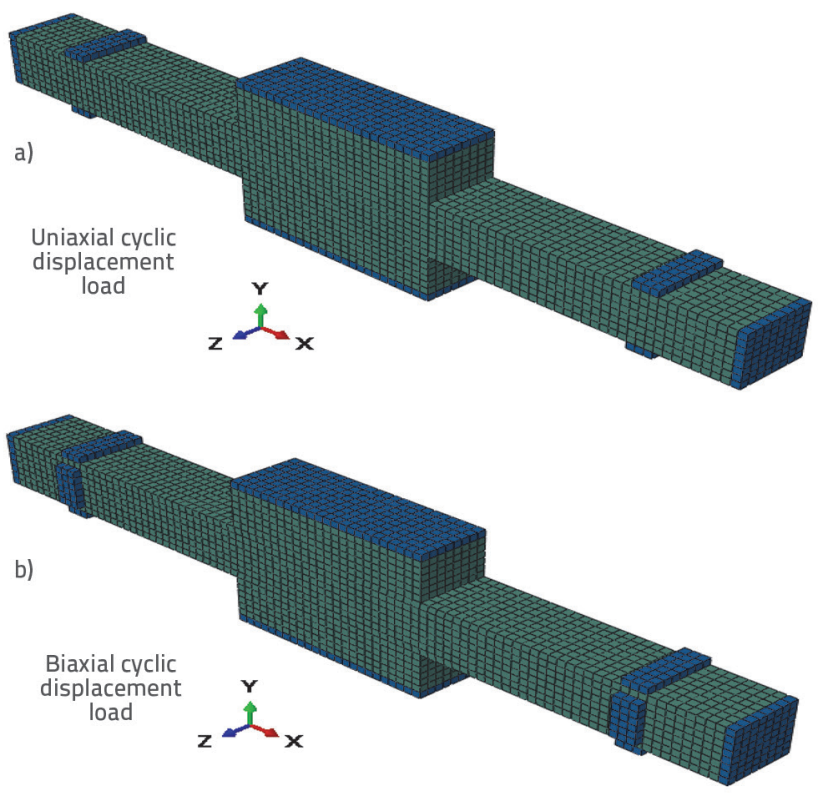

Figure 4. Specimen's mesh: a) uniaxial displacement cyclic load; b) biaxial displacement cyclic load

\section{Validation of numerical modelling}

A comparison of the load-deflection hysteresis obtained from experimental testing of specimen C70-20 [21] and the FE numerical modelling specimen $\mathrm{C} 1$, reveals good correlation with experimental results, as shown in Figure 5. Table 4 shows the ultimate cyclic shear load of column specimen obtained from the numerical model, with the corresponding ultimate deflection and initial stiffness Ki, which also shows good correlation compared with values obtained from experimental analyses [21], with an acceptable error equal to $3 \%, 4.2 \%$ and $-2 \%$ respectively. Figure 6 shows flexural cracks distribution from experimental analysis and damage to concrete (plastic strain) defined from numerical modelling. The plastic strain is concentrated at the bottom of the column 
Table 4. Experimental and numerical results

\begin{tabular}{|c|c|c|c|c|}
\hline State & Specimen designation & $\begin{array}{c}\text { Ultimate shear load } \\
{[\mathrm{kN}]}\end{array}$ & $\begin{array}{c}\text { Ultimate deflection } \\
{[\mathrm{mm}]}\end{array}$ & $\begin{array}{c}\text { Initial stiffness Ki } \\
{[\mathrm{kN} / \mathrm{mm}]}\end{array}$ \\
\hline Experimental & $\mathrm{C} 70-20$ & 51.68 & 38.06 & 11.502 \\
\hline Numerical & $\mathrm{C} 1$ & 53.28 & 39.74 & 11.482 \\
\hline Percentage of error & - & +3.1 & +4.4 & -2 \\
\hline
\end{tabular}

specimen, which is similar to flexural cracks that occurred in the tested specimen C70-20 [21]. The validation between experimental and numerical modelling shows the accuracy of the material model and finite element in simulating the behaviour of HSC columns subjected to uniaxial displacement histories with constant axial load.

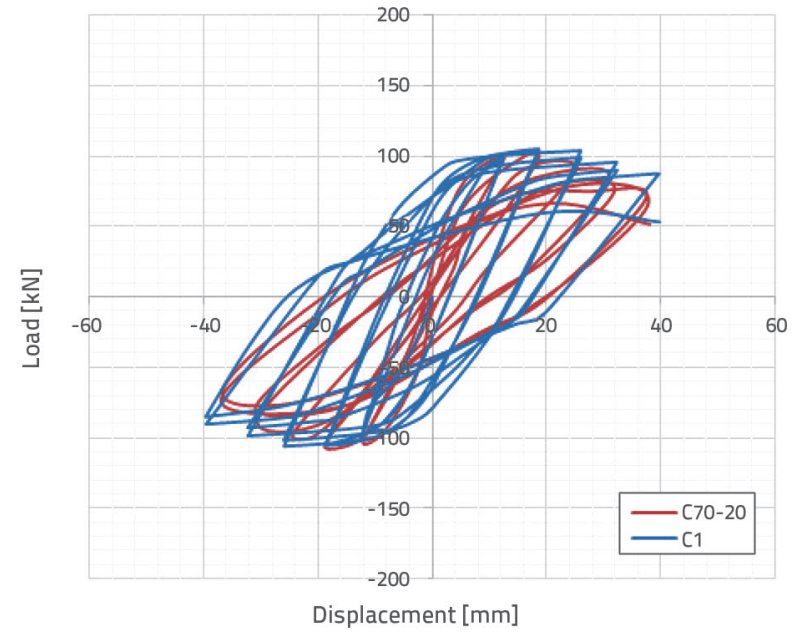

Figure 5. Experimental and numerical load-deflection curve for specimens with $\emptyset 10 \mathrm{~mm}$ stirrups and longitudinal reinforcement ratio $(\rho=0.02)$ under uniaxial load

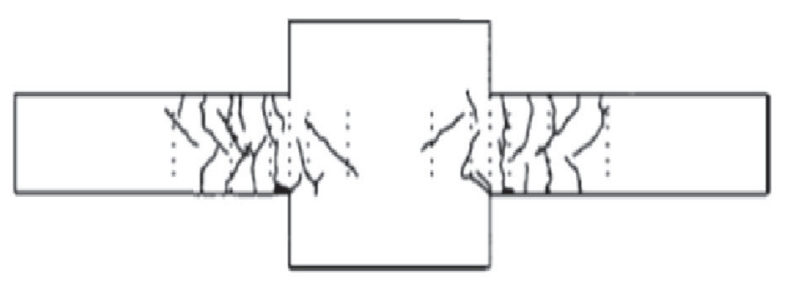

Experimental specimen $\mathrm{C} 70$ - 20
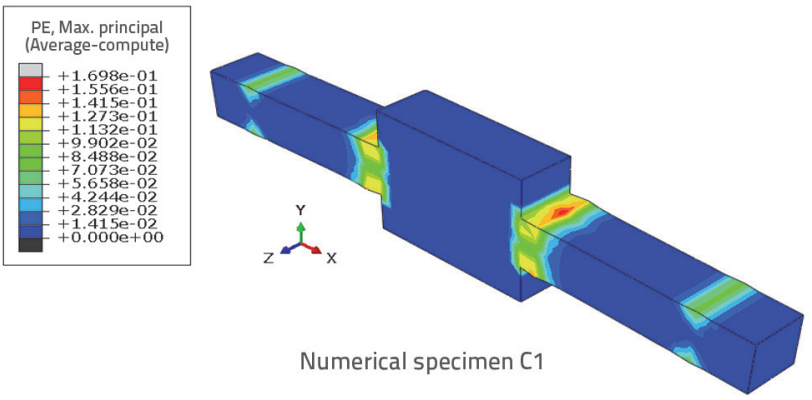

Figure 6. Experimental and numerical damage for specimens with $\emptyset$ $10 \mathrm{~mm}$ stirrups and longitudinal reinforcement ratio ( $\rho=$ 0.02 ) under uniaxial load

Table 5. Details on numerical specimens

\begin{tabular}{|c|c|c|c|c|c|}
\hline No. & Specimen symbol & $\begin{array}{l}\text { Longitudinal reinforcement ratio } \\
\qquad\left(\rho_{1}\right)[\%]\end{array}$ & $\begin{array}{l}\text { *Ash } \\
{\left[\mathrm{mm}^{2}\right]}\end{array}$ & Lateral shear load & $\begin{array}{l}\text { Stirrups tie } \\
{[\mathrm{mm}]}\end{array}$ \\
\hline 1 & ${ }^{* *} \mathrm{C} 1$ & 0.02 & 142.96 & Uniaxial & $\varnothing 10$ \\
\hline 2 & C2 & 0.02 & 254.352 & Uniaxial & $\emptyset 12$ \\
\hline 3 & C3 & 0.02 & 377.17 & Uniaxial & $\emptyset 16$ \\
\hline 4 & C4 & 0.04 & 142.96 & Uniaxial & $\varnothing 10$ \\
\hline 5 & C5 & 0.04 & 254.352 & Uniaxial & $\emptyset 12$ \\
\hline 6 & C6 & 0.04 & 377.17 & Uniaxial & $\varnothing 16$ \\
\hline 7 & C7 & 0.06 & 142.96 & Uniaxial & $\varnothing 10$ \\
\hline 8 & С8 & 0.06 & 254.352 & Uniaxial & $\emptyset 12$ \\
\hline 9 & C9 & 0.06 & 377.17 & Uniaxial & $\varnothing 16$ \\
\hline 10 & C10 & 0.08 & 142.96 & Uniaxial & $\varnothing 10$ \\
\hline 11 & C11 & 0.08 & 254.352 & Uniaxial & $\emptyset 12$ \\
\hline
\end{tabular}


Table 5. Details on numerical specimens - continuation

\begin{tabular}{|c|c|c|c|c|c|}
\hline No. & Specimen symbol & $\begin{array}{l}\text { Longitudinal reinforcement ratio } \\
\qquad\left(\rho_{1}\right)[\%]\end{array}$ & $\begin{array}{l}\text { *Ash } \\
{\left[\mathrm{mm}^{2}\right]}\end{array}$ & Lateral shear load & $\begin{array}{l}\text { Stirrups tie } \\
{[\mathrm{mm}]}\end{array}$ \\
\hline 12 & C12 & 0.08 & 377.17 & Uniaxial & $\varnothing 16$ \\
\hline 13 & C13 & 0.02 & 142.96 & Biaxial & $\varnothing 10$ \\
\hline 14 & C14 & 0.02 & 254.352 & Biaxial & $\varnothing 12$ \\
\hline 15 & C15 & 0.02 & 377.17 & Biaxial & $\varnothing 16$ \\
\hline 16 & C16 & 0.04 & 142.96 & Biaxial & $\varnothing 10$ \\
\hline 17 & C17 & 0.04 & 254.352 & Biaxial & $\varnothing 12$ \\
\hline 18 & C18 & 0.04 & 377.17 & Biaxial & $\varnothing 16$ \\
\hline 19 & C19 & 0.06 & 142.96 & Biaxial & $\varnothing 10$ \\
\hline 20 & C20 & 0.06 & 254.352 & Biaxial & $\emptyset 12$ \\
\hline 21 & C21 & 0.06 & 377.17 & Biaxial & $\varnothing 16$ \\
\hline 22 & C22 & 0.08 & 142.96 & Biaxial & $\varnothing 10$ \\
\hline 23 & C23 & 0.08 & 254.352 & Biaxial & $\varnothing 12$ \\
\hline 24 & C24 & 0.08 & 377.17 & Biaxial & $\varnothing 16$ \\
\hline
\end{tabular}

\section{Numerical parametric study}

In the present study, four parameters were considered to develop the control numerical validated specimen $\mathrm{C}$. These parameters are: longitudinal reinforcement ratio, total cross-sectional area of confinement steel (Ash), and the uniaxial and biaxial cyclic shear load. The displacement history considered for the uniaxial and biaxial cyclic shear load is the same as the one previously presented in Figure 2. Details on numerical specimens are given in Table 5. All specimens were subjected to constant axial load similar to that applied to the experimental specimen C70-20 [21].

\section{Numerical results}

Numerical results are presented based on the finite element model of twenty-four specimens with the considered parametric study as mentioned earlier in the previous section. Load-deflection hysteretic curves of specimens are shown in figures 7 to 12, while the data obtained from curves are summarized in Table 6. The damage to concrete (plastic strain) is presented in figures 13 to 18 . Comparison of specimen results are shown in tables 7 and 8 and in figures 19 to 22 .

Table 6. Numerical results for all specimens

\begin{tabular}{|c|c|c|c|c|c|c|c|c|c|c|}
\hline Specimen & $\rho$ & $\begin{array}{c}\text { Ash } \\
{\left[\mathrm{mm}^{2}\right]}\end{array}$ & $\begin{array}{l}\text { Stirrup } \\
\text { diameter } \\
\text { [mm] }\end{array}$ & $\begin{array}{l}\text { Total } \\
\text { No. of } \\
\text { cycles }\end{array}$ & $\begin{array}{l}\text { Maximum } \\
\text { load } \\
{[\mathrm{kN}]}\end{array}$ & $\begin{array}{c}\text { Deflection at } \\
\text { maximum load } \\
{[\mathrm{mm}]}\end{array}$ & $\begin{array}{c}\text { Cycle at } \\
\text { maximum load }\end{array}$ & $\begin{array}{c}\text { Ultimate } \\
\text { load } \\
{[\mathrm{kN}]}\end{array}$ & $\begin{array}{c}\text { Ultimate } \\
\text { deflection } \\
{[\mathrm{mm}]}\end{array}$ & $\begin{array}{c}\text { Initial } \\
\text { stiffness } \\
\mathbf{K i} \\
{[\mathrm{kN} / \mathrm{mm}]}\end{array}$ \\
\hline C1 & 0.02 & 142.96 & $\varnothing 10$ & 21 & 106.02 & 25.6 & 9 & 53.28 & 39.74 & 11.482 \\
\hline$C 2$ & 0.02 & 254.352 & $\emptyset 12$ & 27 & 109.0 & 25.83 & 9 & 92.04 & 52.1 & 11.684 \\
\hline C3 & 0.02 & 377.17 & $\emptyset 16$ & 30 & 113.67 & 25.9 & 9 & 94.21 & 58.27 & 11.760 \\
\hline C4 & 0.04 & 142.96 & $\varnothing 10$ & 23 & 137.57 & 25.9 & 9 & 123.4 & 48.33 & 13.354 \\
\hline C5 & 0.04 & 254.352 & $\emptyset 12$ & 27 & 140.43 & 26.2 & 9 & 132.04 & 55.02 & 13.405 \\
\hline C6 & 0.04 & 377.17 & $\emptyset 16$ & 27 & 144.53 & 26.22 & 9 & 135.07 & 54.88 & 13.506 \\
\hline C7 & 0.06 & 142.96 & $\varnothing 10$ & 27 & 154.01 & 26.8 & 10 & 163.02 & 53.85 & 15.582 \\
\hline C8 & 0.06 & 254.352 & $\emptyset 12$ & 26 & 172.52 & 27.6 & 9 & 168.58 & 54.29 & 15.658 \\
\hline C9 & 0.06 & 377.17 & $\emptyset 16$ & 34 & 179.31 & 28.51 & 29 & 179.2 & 60.35 & 15.747 \\
\hline C10 & 0.08 & 142.96 & $\varnothing 10$ & 25 & 167.98 & 25.12 & 5 & 125.12 & 45.23 & 17.795 \\
\hline C11 & 0.08 & 254.352 & $\emptyset 12$ & 27 & 190.5 & 25.2 & 9 & 169.33 & 51.69 & 17.846 \\
\hline C12 & 0.08 & 377.17 & $\varnothing 16$ & 25 & 207.37 & 26.1 & 10 & 203.81 & 47.65 & 17.926 \\
\hline C13 & 0.02 & 142.96 & $\varnothing 10$ & 14 & 60.94 & 18.59 & 5 & 55.33 & 32.06 & 9.601 \\
\hline C14 & 0.02 & 254.352 & $\emptyset 12$ & 20 & 60.7 & 18.57 & 5 & 51.36 & 39.35 & 9.695 \\
\hline
\end{tabular}


Table 6. Numerical results for all specimens - continuation

\begin{tabular}{|c|c|c|c|c|c|c|c|c|c|c|}
\hline Specimen & $\rho$ & $\begin{array}{c}\text { Ash } \\
{\left[\mathrm{mm}^{2}\right]}\end{array}$ & $\begin{array}{c}\text { Stirrup } \\
\text { diameter } \\
{[\mathrm{mm}]}\end{array}$ & $\begin{array}{l}\text { Total } \\
\text { No. of } \\
\text { cycles }\end{array}$ & $\begin{array}{c}\text { Maximum } \\
\text { load } \\
{[\mathrm{kN}]}\end{array}$ & $\begin{array}{c}\text { Deflection at } \\
\text { maximum load } \\
{[\mathrm{mm}]}\end{array}$ & $\begin{array}{c}\text { Cycle at } \\
\text { maximum load }\end{array}$ & $\begin{array}{c}\text { Ultimate } \\
\text { load } \\
{[\mathrm{kN}]}\end{array}$ & $\begin{array}{c}\text { Ultimate } \\
\text { deflection } \\
{[\mathrm{mm}]}\end{array}$ & $\begin{array}{c}\text { Initial } \\
\text { stiffness } \\
\text { Ki } \\
{[\mathrm{kN} / \mathrm{mm}]}\end{array}$ \\
\hline C15 & 0.02 & 377.17 & $\emptyset 16$ & 19 & 61.7 & 18.68 & 6 & 56 & 39.53 & 9.866 \\
\hline C16 & 0.04 & 142.96 & $\varnothing 10$ & 12 & 77.88 & 25.01 & 6 & 77.78 & 25.6 & 12.358 \\
\hline C17 & 0.04 & 254.352 & $\emptyset 12$ & 14 & 79.44 & 25.61 & 9 & 78.53 & 31.68 & 12.458 \\
\hline C18 & 0.04 & 377.17 & $\emptyset 16$ & 10 & 80.5 & 25.7 .7 & 6 & 81.3 & 31.72 & 12.536 \\
\hline C19 & 0.06 & 142.96 & $\varnothing 10$ & 12 & 92.72 & 25.47 & 9 & 90.4 & 25.5 & 14.210 \\
\hline C2O & 0.06 & 254.352 & $\emptyset 12$ & 12 & 94.88 & 25.5 & 9 & 93.05 & 25.5 & 14.346 \\
\hline C21 & 0.06 & 377.17 & $\emptyset 16$ & 11 & 97.25 & 25.9 & 10 & 97.25 & 25.9 & 14.466 \\
\hline$C 22$ & 0.08 & 142.96 & $\emptyset 10$ & 12 & 96.61 & 24.26 & 6 & 90.8 & 25.25 & 16.267 \\
\hline C23 & 0.08 & 254.352 & $\emptyset 12$ & 12 & 98.88 & 25.25 & 9 & 96.1 & 25.3 & 16.293 \\
\hline C24 & 0.08 & 377.17 & $\emptyset 16$ & 10 & 100.01 & 26.33 & 6 & 99.97 & 25.26 & 16.372 \\
\hline
\end{tabular}

Table 7. Ratio increment in load and deflection

\begin{tabular}{|c|c|c|c|c|c|c|c|c|c|c|c|c|}
\hline $\begin{array}{l}\text { 들 } \\
\text { ڤัँ }\end{array}$ & $\rho$ & $\begin{array}{c}\text { Ash } \\
{\left[\mathrm{mm}^{2}\right]}\end{array}$ & $\begin{array}{c}\text { Maximum } \\
\text { load } \\
{[\mathrm{kN}]}\end{array}$ & $\begin{array}{c}\text { Increment } \\
\text { ratio at } \\
\text { maximum } \\
\text { load } \\
{[\%]}\end{array}$ & $\begin{array}{c}\text { Deflection } \\
\text { at maximum } \\
\text { load } \\
{[\mathrm{mm}]}\end{array}$ & $\begin{array}{l}\text { Increment } \\
\text { ratio for } \\
\text { Deflection } \\
\text { at maximum } \\
\text { load [\%] }\end{array}$ & $\begin{array}{c}\text { Ultimate } \\
\text { load } \\
{[\mathrm{kN}]}\end{array}$ & $\begin{array}{c}\text { Increment } \\
\text { ratio at } \\
\text { ultimate } \\
\text { load } \\
{[\%]}\end{array}$ & $\begin{array}{c}\text { Ultimate } \\
\text { deflection } \\
\text { [mm] }\end{array}$ & $\begin{array}{c}\text { Increment } \\
\text { ratio at } \\
\text { ultimate } \\
\text { deflection } \\
{[\%]}\end{array}$ & $\begin{array}{c}\text { Initial } \\
\text { stiffness } \\
\mathbf{K i} \\
{[\mathrm{kN} / \mathrm{mm}]}\end{array}$ & $\begin{array}{c}\text { Increment } \\
\text { ratio at } \\
\text { initial } \\
\text { stiffness } \\
{[\%]}\end{array}$ \\
\hline C1 & 0.02 & 142.96 & 106.02 & \multirow{3}{*}{7.2} & 25.6 & \multirow{3}{*}{1.1} & 53.28 & \multirow{3}{*}{77} & 39.74 & \multirow{3}{*}{46} & 11.482 & \multirow{3}{*}{0.2} \\
\hline C2 & 0.02 & 254.352 & 109.0 & & 25.83 & & 92.04 & & 52.1 & & 11.684 & \\
\hline C3 & 0.02 & 377.17 & 113.67 & & 25.9 & & 94.21 & & 58.27 & & 11.760 & \\
\hline C4 & 0.04 & 142.96 & 137.57 & \multirow{3}{*}{5.5} & 25.9 & \multirow{3}{*}{1.2} & 123.4 & \multirow{3}{*}{9.75} & 48.33 & \multirow{3}{*}{12.5} & 13.354 & \multirow{3}{*}{0.2} \\
\hline C5 & 0.04 & 254.352 & 140.43 & & 26.2 & & 132.04 & & 55.02 & & 13.405 & \\
\hline C6 & 0.04 & 377.17 & 144.53 & & 26.22 & & 135.07 & & 54.88 & & 13.506 & \\
\hline C7 & 0.06 & 142.96 & 154.01 & \multirow{3}{*}{16.5} & 26.8 & \multirow{3}{*}{6.3} & 163.02 & \multirow{3}{*}{10} & 53.85 & \multirow{3}{*}{13} & 15.582 & \multirow{3}{*}{0.2} \\
\hline C8 & 0.06 & 254.352 & 172.52 & & 27.6 & & 168.58 & & 54.29 & & 15.658 & \\
\hline C9 & 0.06 & 377.17 & 179.31 & & 28.51 & & 179.2 & & 60.35 & & 15.747 & \\
\hline C10 & 0.08 & 142.96 & 167.98 & \multirow{3}{*}{24} & 25.12 & \multirow{3}{*}{4} & 160.12 & \multirow{3}{*}{27} & 45.23 & \multirow{3}{*}{4.4} & 17.795 & \multirow{3}{*}{0.2} \\
\hline C11 & 0.08 & 254.352 & 190.5 & & 25.2 & & 169.33 & & 51.69 & & 17.846 & \\
\hline C12 & 0.08 & 377.17 & 207.37 & & 26.1 & & 203.81 & & 47.65 & & 17.926 & \\
\hline C13 & 0.02 & 142.96 & 60.94 & \multirow{3}{*}{1.2} & 18.59 & \multirow{3}{*}{0.5} & 55.33 & \multirow{3}{*}{1.2} & 32.06 & \multirow{3}{*}{21} & 9.601 & \multirow{3}{*}{0.2} \\
\hline C14 & 0.02 & 254.352 & 60.7 & & 18.57 & & 51.36 & & 39.35 & & 9.695 & \\
\hline C15 & 0.02 & 377.17 & 61.7 & & 18.68 & & 56 & & 39.53 & & 9.866 & \\
\hline C16 & 0.04 & 142.96 & 77.88 & \multirow{3}{*}{3.9} & 25.01 & \multirow{3}{*}{2} & 77.78 & \multirow{3}{*}{4.5} & 25.6 & \multirow{3}{*}{24} & 12.358 & \multirow{3}{*}{0.2} \\
\hline C17 & 0.04 & 254.352 & 79.44 & & 25.61 & & 78.53 & & 31.68 & & 12.458 & \\
\hline C18 & 0.04 & 377.17 & 80.5 & & 25.7 .7 & & 81.3 & & 31.72 & & 12.536 & \\
\hline C19 & 0.06 & 142.96 & 92.72 & & 25.47 & & 90.4 & & 25.5 & & 14.210 & \\
\hline C20 & 0.06 & 254.352 & 94.88 & 5.4 & 25.5 & 1.6 & 93.05 & 7.5 & 25.5 & 1 & 14.346 & 0.2 \\
\hline C21 & 0.06 & 377.17 & 97.25 & & 25.9 & & 97.25 & & 25.9 & & 14.466 & \\
\hline C22 & 0.08 & 142.96 & 96.61 & & 24.26 & & 90.8 & & 25.25 & & 16.267 & \\
\hline C23 & 0.08 & 254.352 & 98.88 & 4 & 25.25 & 8.5 & 96.1 & 10 & 25.3 & 1 & 16.293 & 0.2 \\
\hline C24 & 0.08 & 377.17 & 100.01 & & 26.33 & & 99.97 & & 25.26 & & 16.372 & \\
\hline
\end{tabular}


Table 8. Ratio of load and deflection for biaxial and uniaxial load

\begin{tabular}{|c|c|c|c|c|}
\hline Biaxial / uniaxial sample & Percentage of max. load & $\begin{array}{c}\text { Percentage of deflection } \\
\text { at max. load }\end{array}$ & $\begin{array}{l}\text { Percentage of ultimate } \\
\text { load }\end{array}$ & $\begin{array}{c}\text { Percentage of ultimate } \\
\text { deflection }\end{array}$ \\
\hline C13/C1 & 57.5 & 72.6 & 103.8 & 80.7 \\
\hline C14/C2 & 55.7 & 71.9 & 55.8 & 75.5 \\
\hline C15/C3 & 54.3 & 72.1 & 59.4 & 67.8 \\
\hline C16/C4 & 56.6 & 96.6 & 63.0 & 53.0 \\
\hline $\mathrm{C} 17 / \mathrm{C5}$ & 56.6 & 97.7 & 59.5 & 57.6 \\
\hline C18/C6 & 55.7 & 98.0 & 60.2 & 57.8 \\
\hline C19/C7 & 60.2 & 95.0 & 55.5 & 47.4 \\
\hline C20/C8 & 55.0 & 92.4 & 55.2 & 47.0 \\
\hline $\mathrm{C} 21 / \mathrm{C9}$ & 54.2 & 90.8 & 54.3 & 38.5 \\
\hline C22/C10 & 57.5 & 96.6 & 72.6 & 55.8 \\
\hline C23/C11 & 51.9 & 100.2 & 56.8 & 48.9 \\
\hline C24/C12 & 48.2 & 100.9 & 49.1 & 53.0 \\
\hline
\end{tabular}

a)

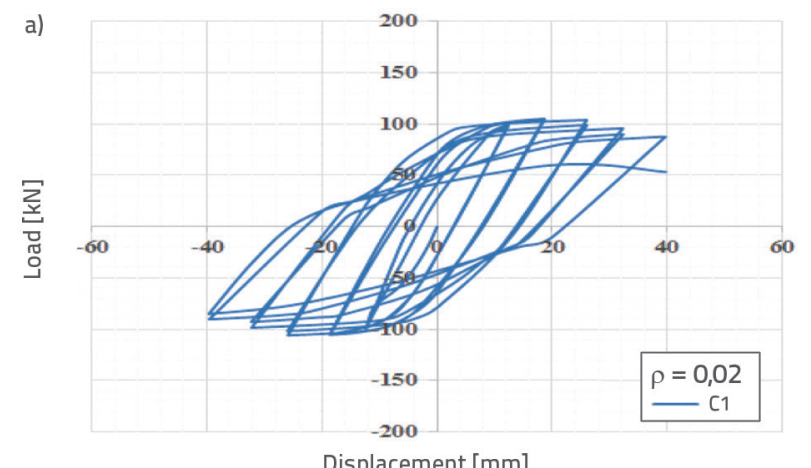

Displacement [mm]

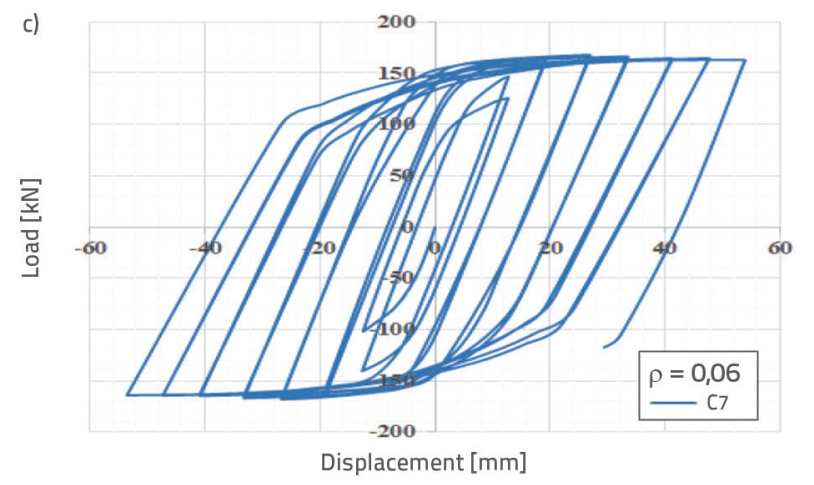

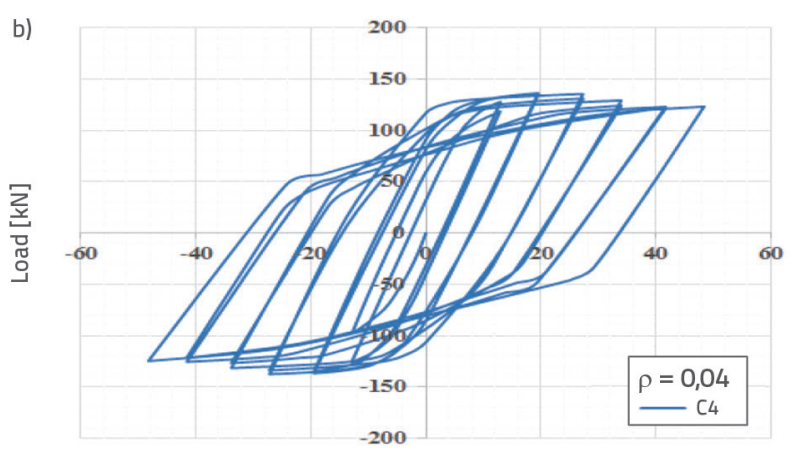

Displacement [mm]

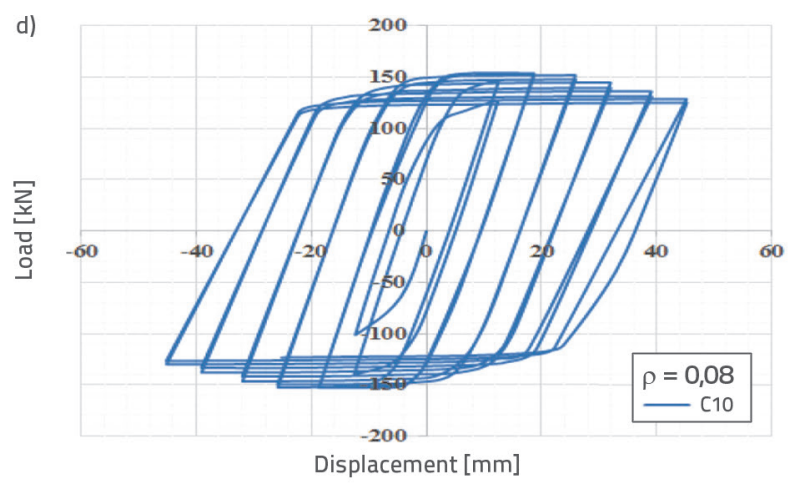

Figure 7. Numerical load-deflection curves for specimen with $\emptyset 10 \mathrm{~mm}$ stirrups under uniaxial load: a) with $\rho=0.02$; b) with $\rho=0.04$; c) with $\rho=0.06$; d) with $\rho=0.08$ ( $\rho$ - longitudinal reinforcement coefficient) 


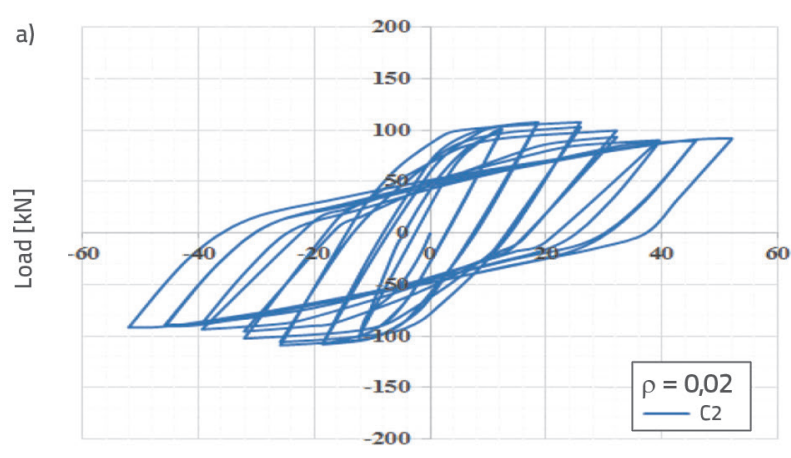

Displacement [mm]

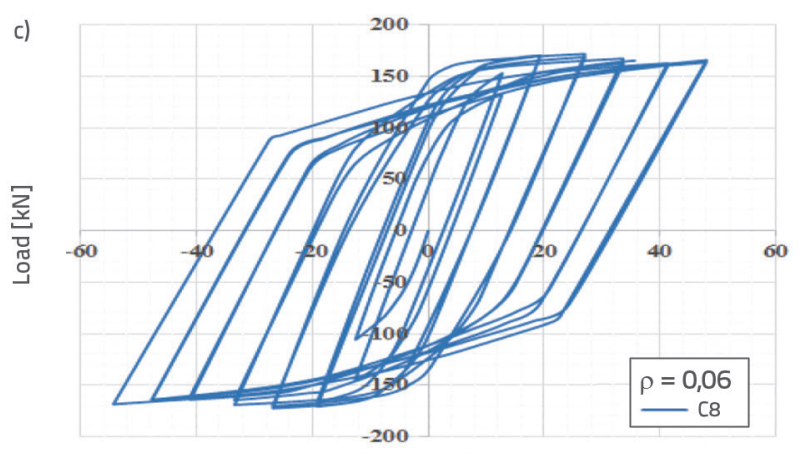

Displacement [mm]

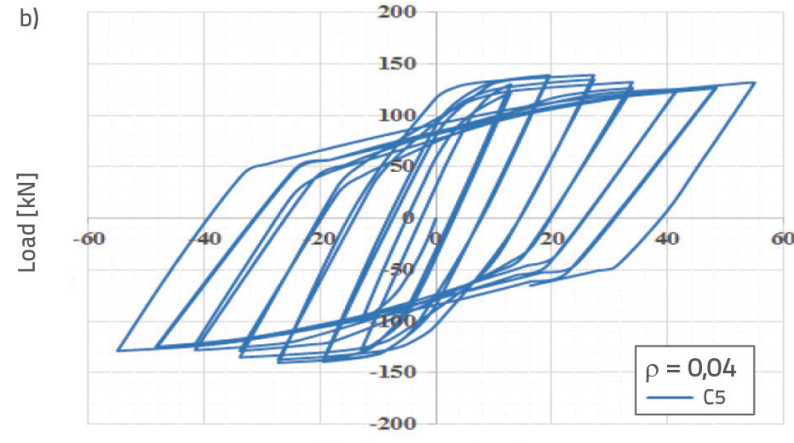

Displacement [mm]

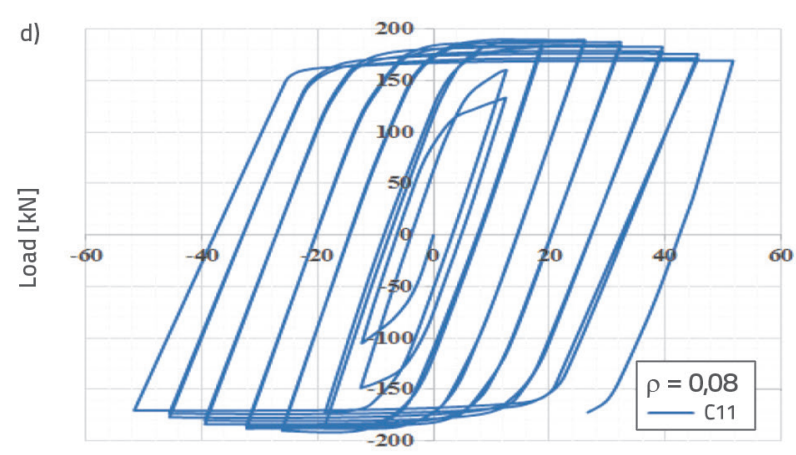

Displacement [mm]

Figure 8. Numerical load-deflection curves for specimen with $\emptyset 12 \mathrm{~mm}$ stirrups under uniaxial load: a) with $\rho=0.02$; b) with $\rho=0.04$; c) with $\rho=0.06$; d) with $\rho=0.08$ ( $\rho$ - longitudinal reinforcement coefficient)

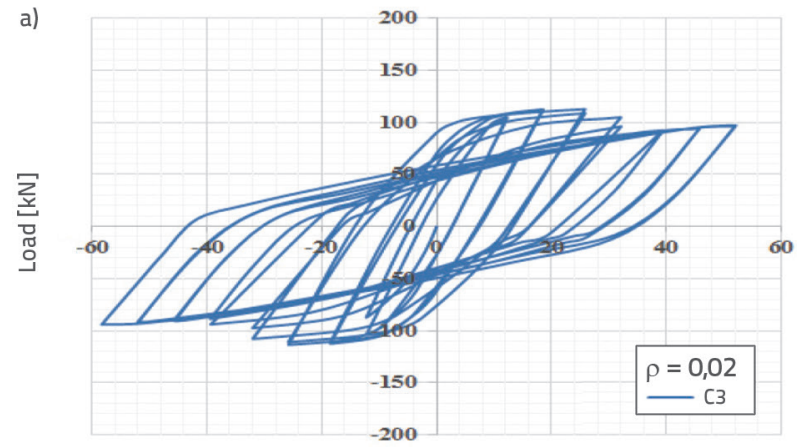

Displacement [mm]

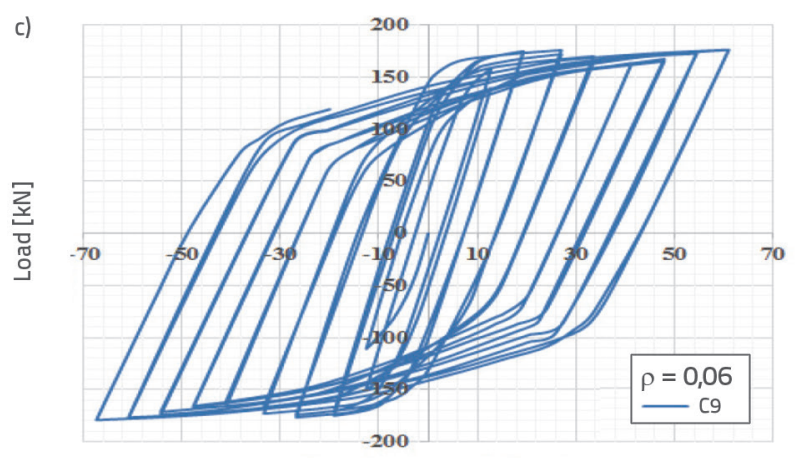

Displacement [mm]

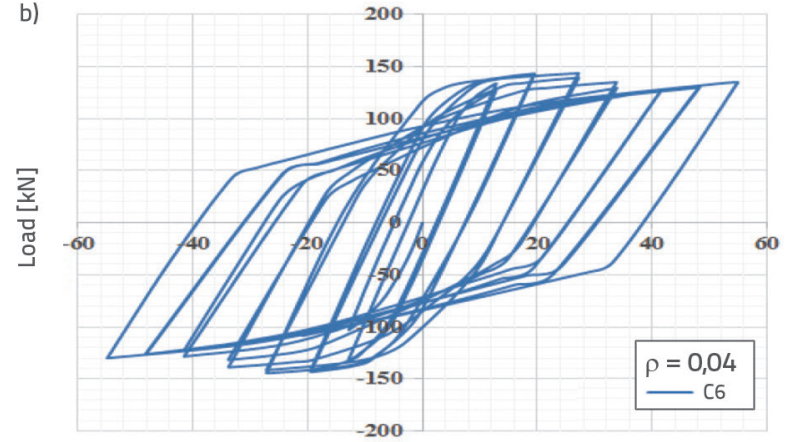

Displacement [mm]

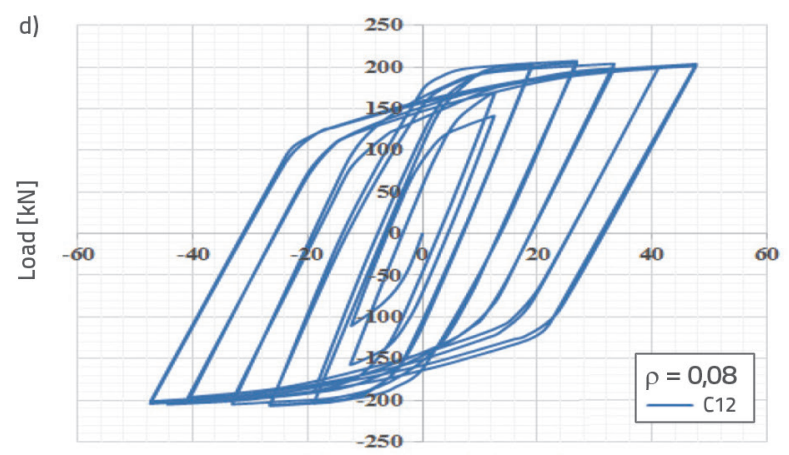

Displacement [mm]

Figure 9. Numerical load-deflection curves for specimen with $\emptyset 16 \mathrm{~mm}$ stirrups under uniaxial load: a) with $\rho=0.02$; b) with $\rho=0.04$; c) with $\rho=0.06$; d) with $\rho=0.08$ ( $\rho$ - longitudinal reinforcement coefficient) 
a)

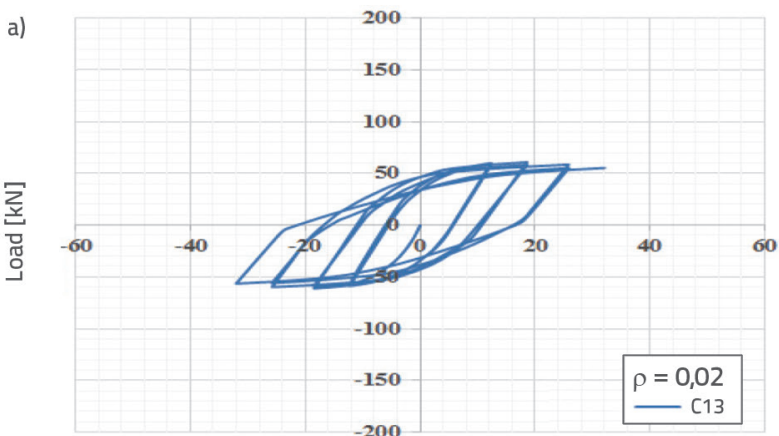

Displacement [mm]

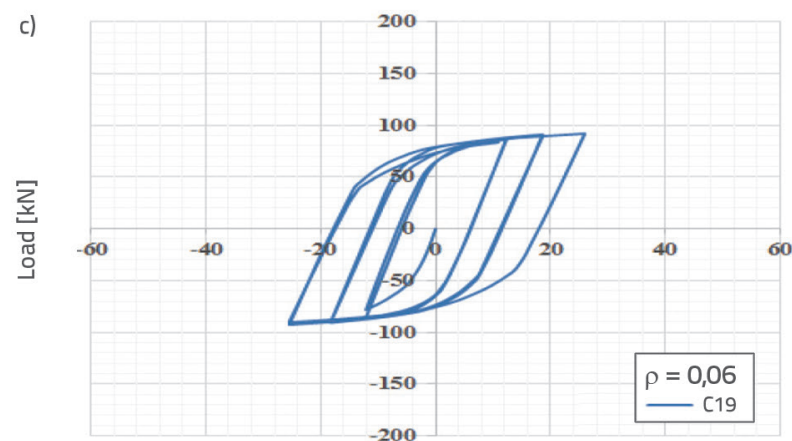

Displacement $[\mathrm{mm}]$

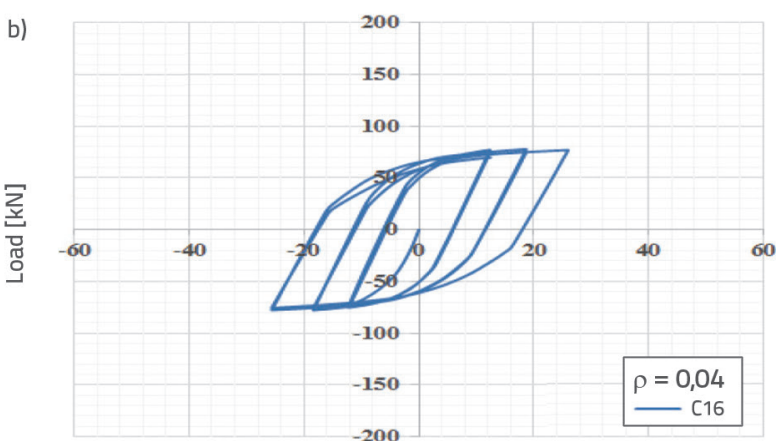

Displacement $[\mathrm{mm}]$

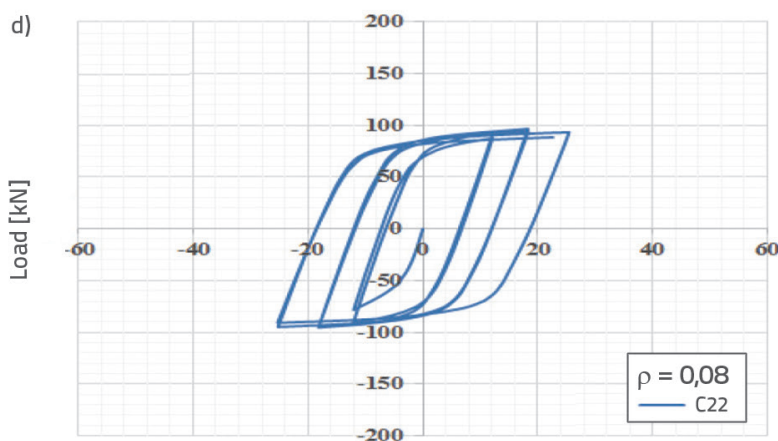

Displacement $[\mathrm{mm}]$

Figure 10. Numerical load-deflection curves for specimens with $\emptyset 10 \mathrm{~mm}$ stirrups under biaxial load: a) with $\rho=0.02$; b) with $\rho=0.04$; c) with $\rho=\mathbf{0 . 0 6}$; d) with $\rho=\mathbf{0 . 0 8}(\rho$ - longitudinal reinforcement coefficient)

a)

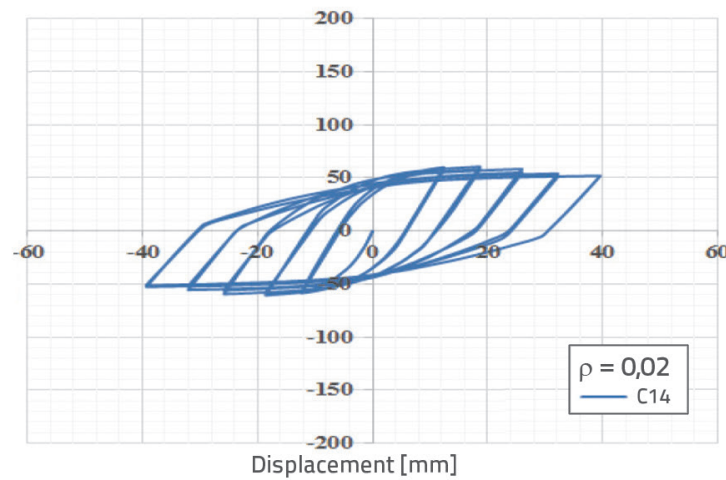

c)

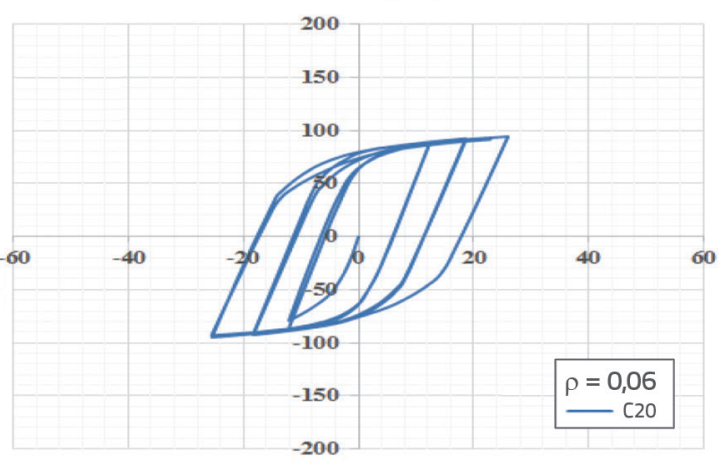

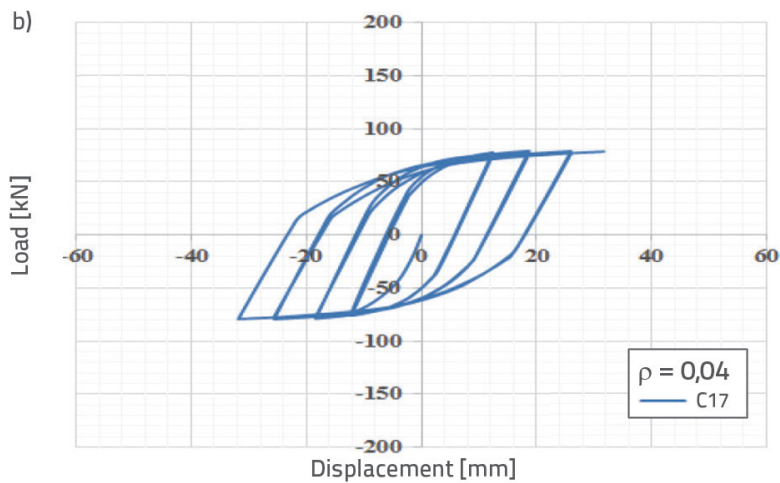

d)

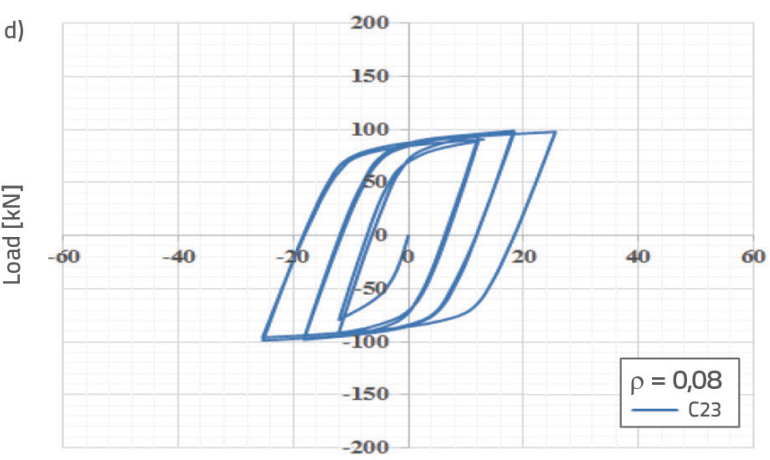

Displacement $[\mathrm{mm}]$

Figure 11. Numerical load-deflection curves for specimens with $\emptyset 12 \mathrm{~mm}$ stirrups under biaxial load: a) with $\rho=0.02$; b) with $\rho=0.04$; c) with $\rho=0.06$; d) with $\rho=0.08$ ( $\rho$ - longitudinal reinforcement coefficient) 

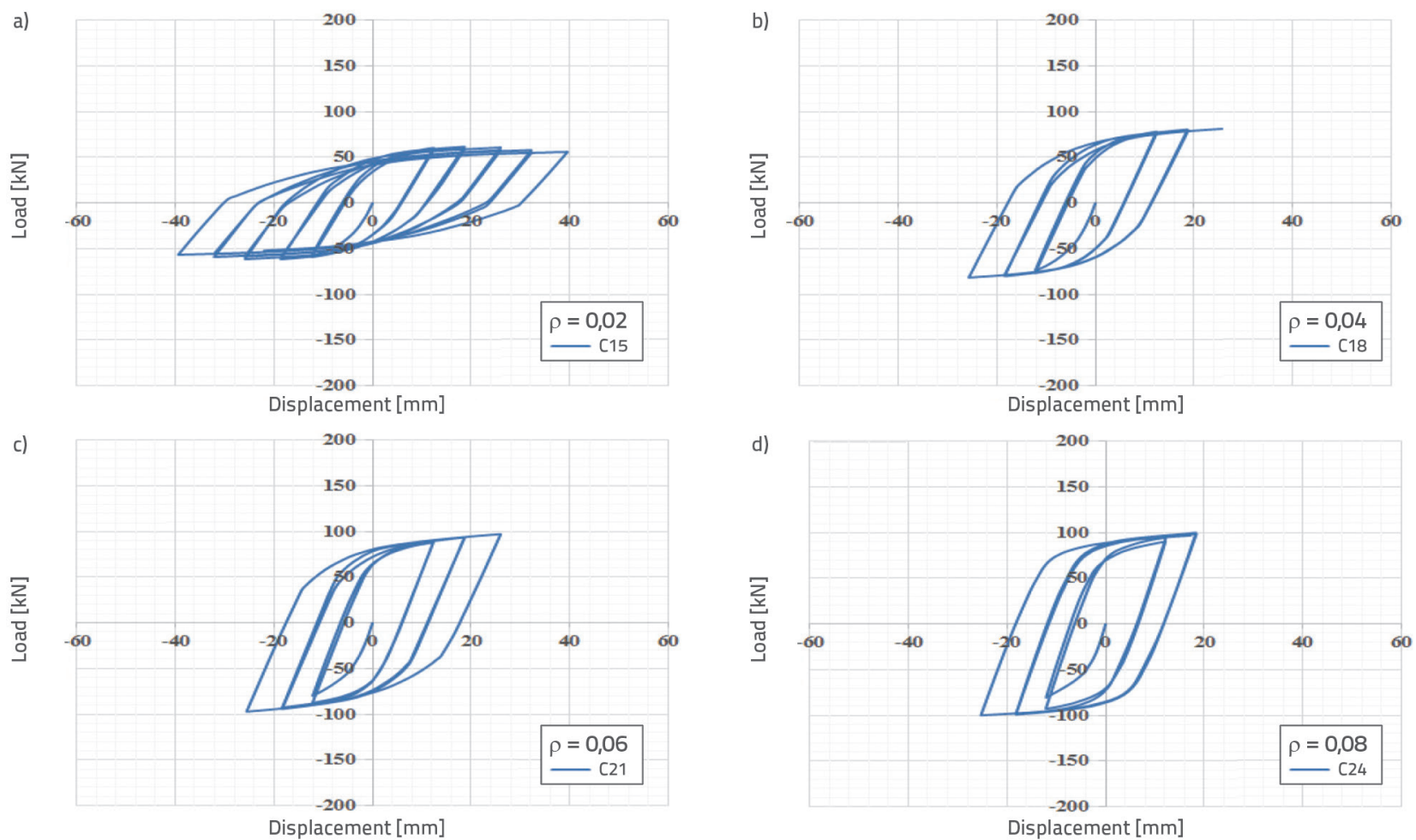

Figure 12. Numerical load-deflection curves for specimens with $\emptyset 16 \mathrm{~mm}$ stirrups under biaxial load: a) with $\rho=0.02$; b) with $\rho=0.04$; c) with $\rho=0.06$; d) with $\rho=0.08$ ( $\rho$ - longitudinal reinforcement coefficient)
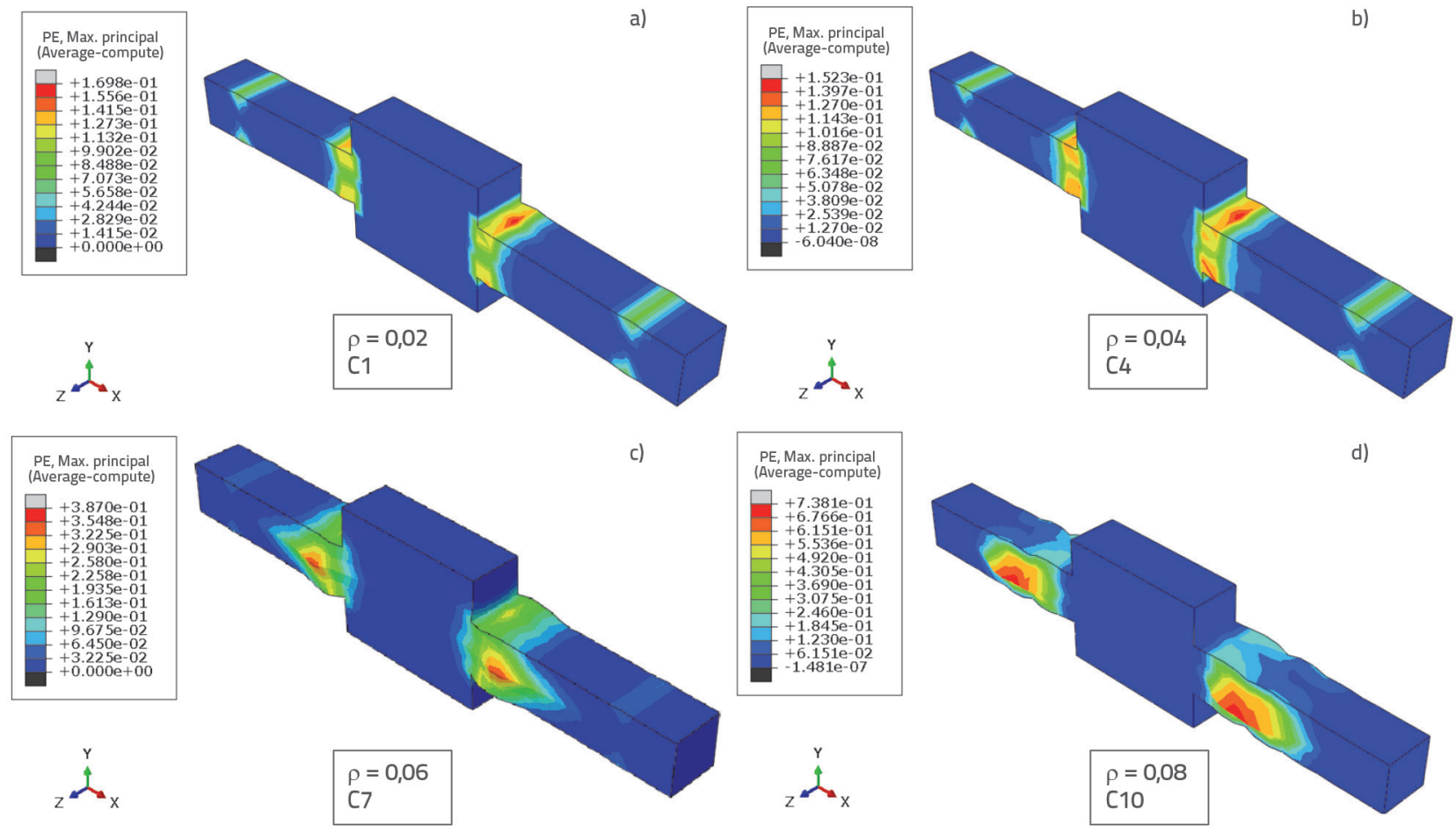

d)

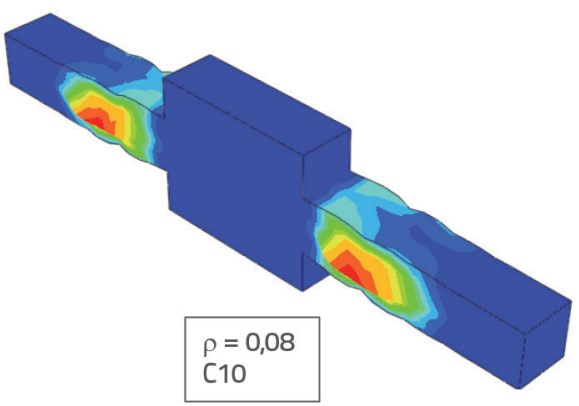

Figure 13. Concrete plastic strain (damage) for specimens with $\emptyset 10 \mathrm{~mm}$ stirrups under uniaxial load: a) with $\rho=0.02$; b) with $\rho=0.04$; c) with $\rho=0.06$; d) with $\rho=0.08$ ( $\rho$ - longitudinal reinforcement coefficient) 

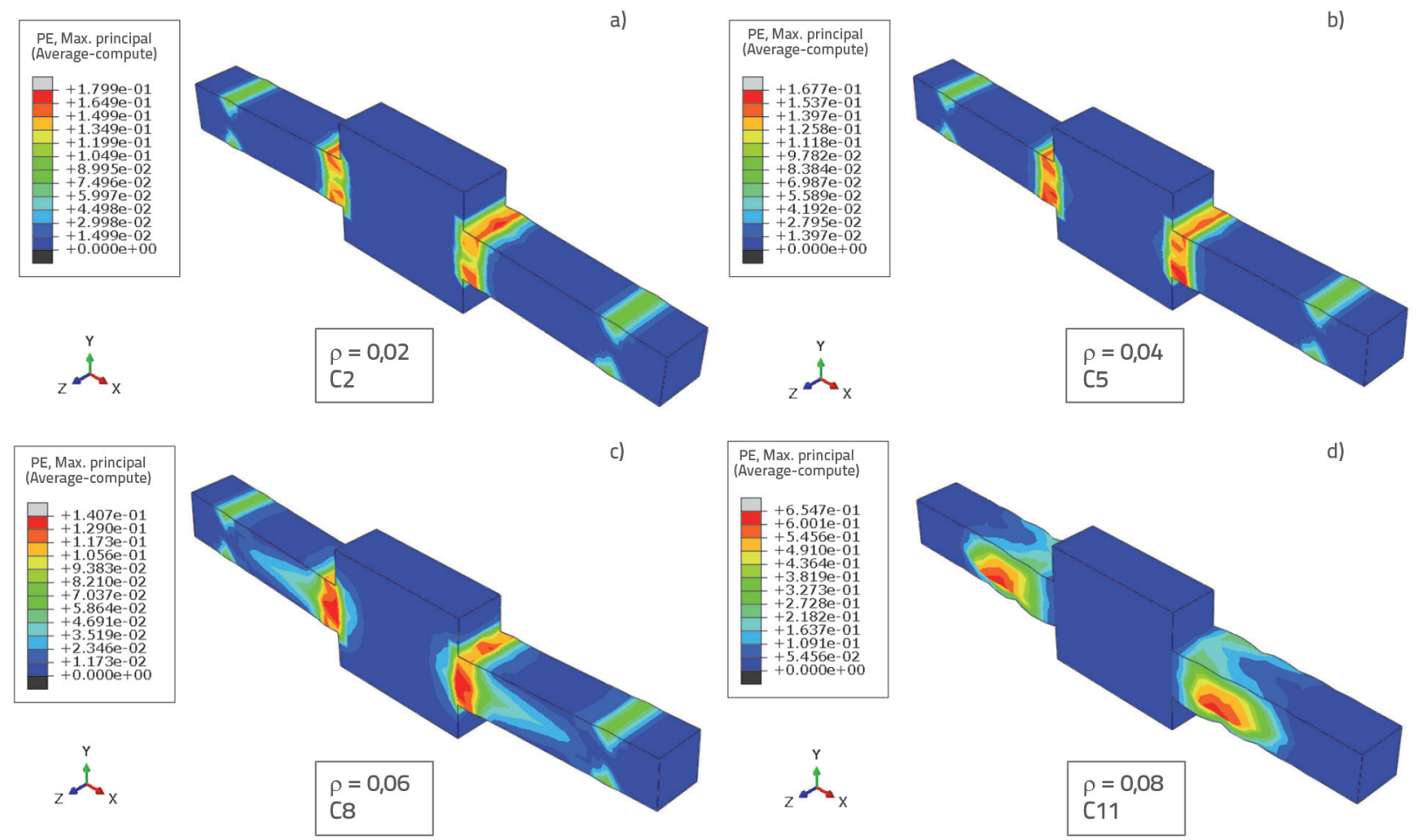

d)<smiles>[Z]C([Y])[3H]</smiles>

Figure 14. Concrete plastic strain (damage) for specimens with $\emptyset 12 \mathrm{~mm}$ stirrups under uniaxial load: a) with $\rho=0.02$; b) with $\rho=0.04$; c) with $\rho=0.06$; d) with $\rho=0.08$ ( $\rho$ - longitudinal reinforcement coefficient)
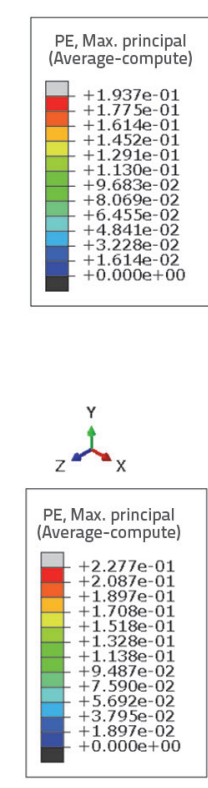

$z^{y}$
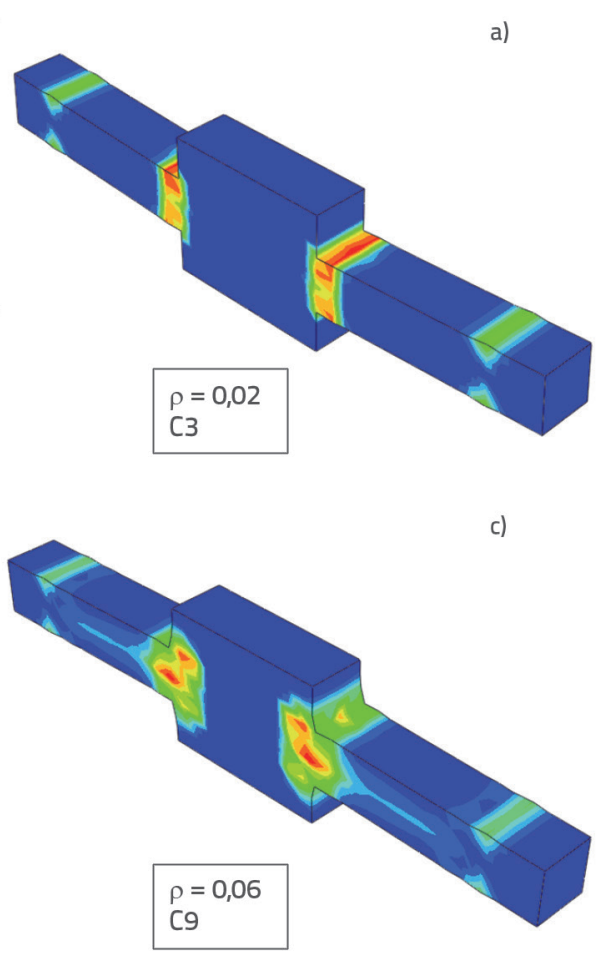
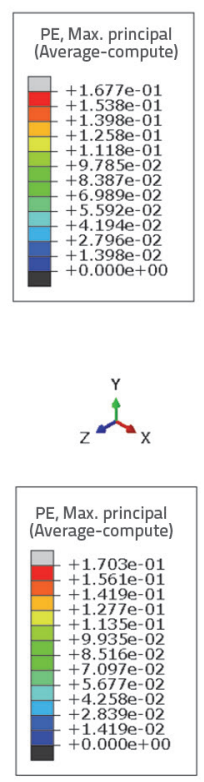

$z \stackrel{x}{ }$ b)

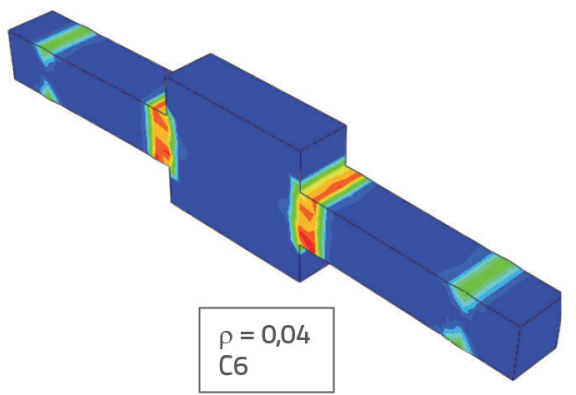

d)

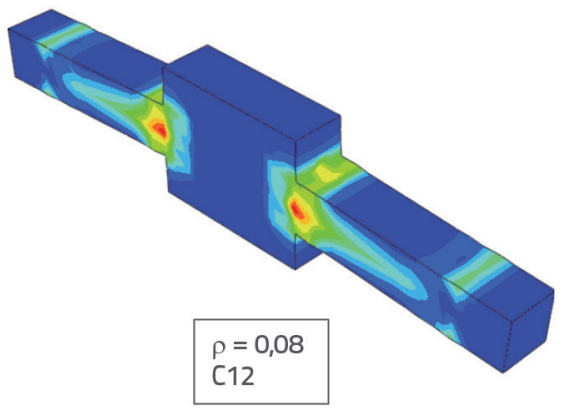

Figure 15. Concrete plastic strain (damage) for specimens with $\emptyset 16 \mathrm{~mm}$ stirrups under uniaxial load: a) with $\rho=0.02$; b) with $\rho=0.04$; c) with $\rho=0.06$; d) with $\rho=0.08$ ( $\rho$ - longitudinal reinforcement coefficient) 

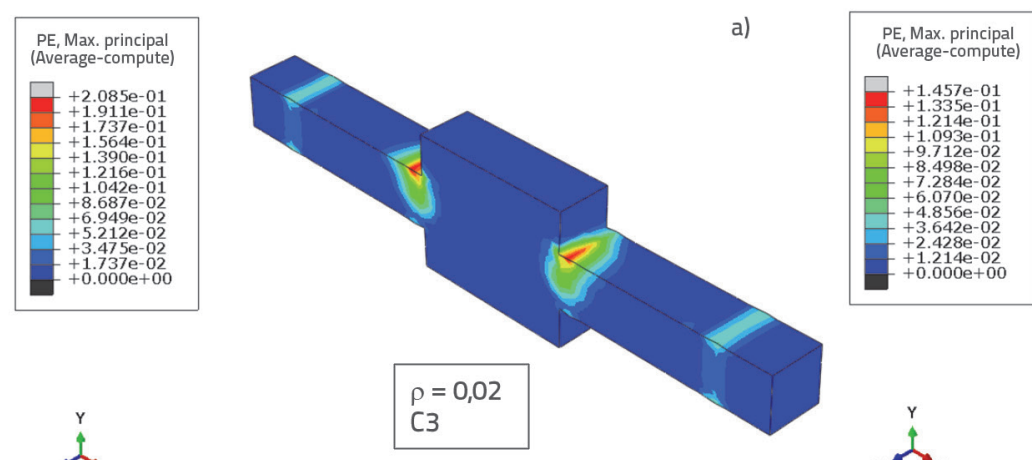

b)
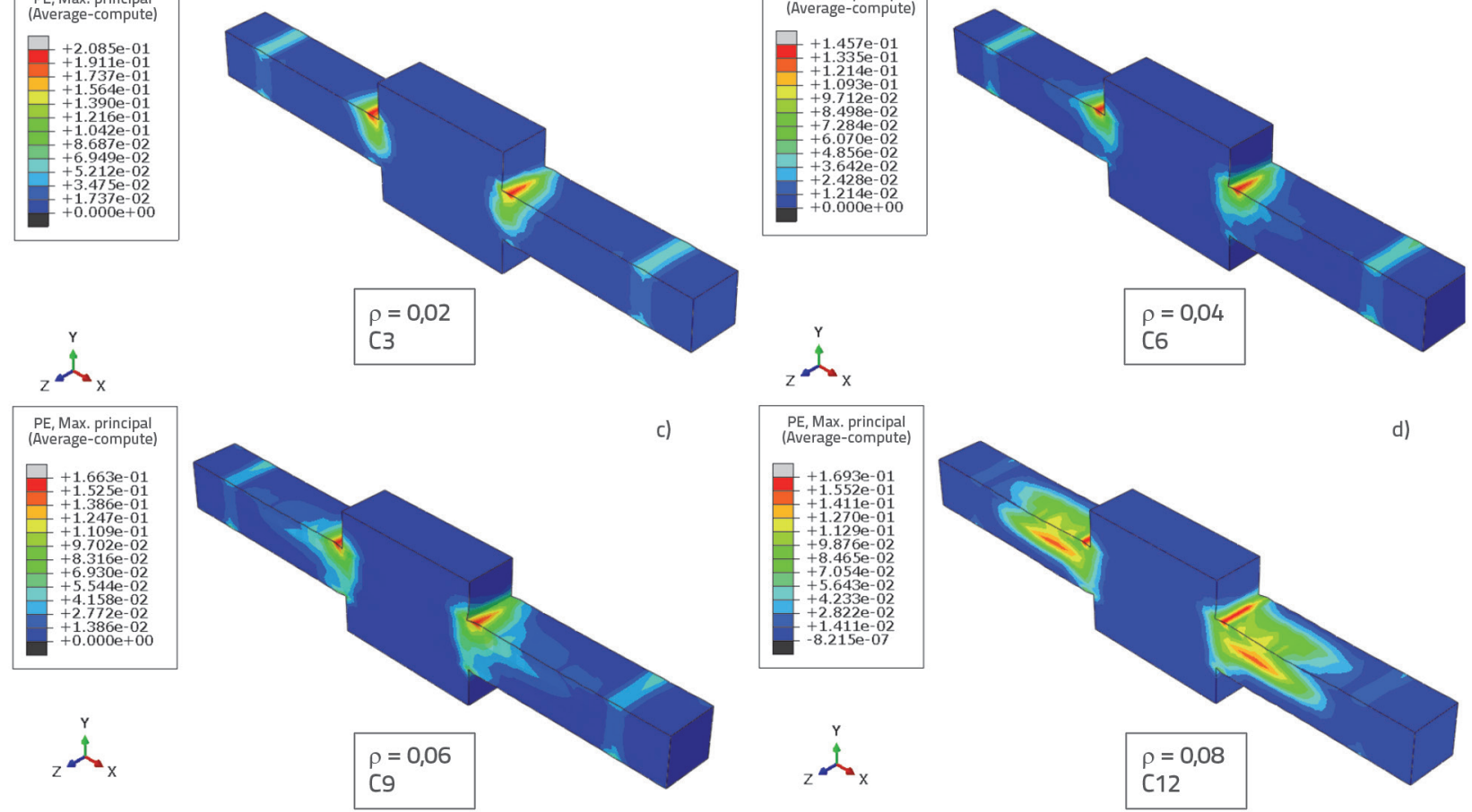

$\stackrel{\text { Y }}{\text { L }}$
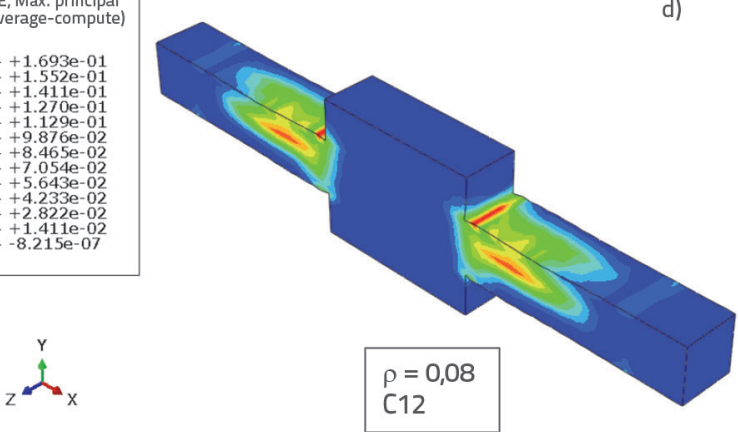

Figure 16. Concrete plastic strain (damage) for specimens with $\emptyset 10 \mathrm{~mm}$ stirrups under biaxial load: a) with $\rho=0.02$; b) with $\rho=0.04$; c) with $\rho=0.06$; d) with $\rho=0.08$ ( $\rho$ - longitudinal reinforcement coefficient)

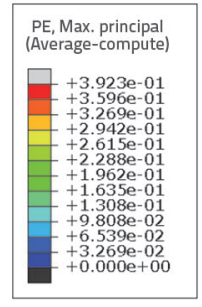

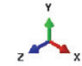

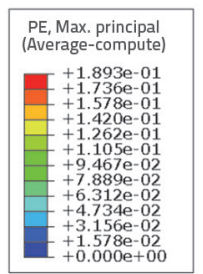

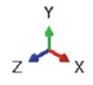

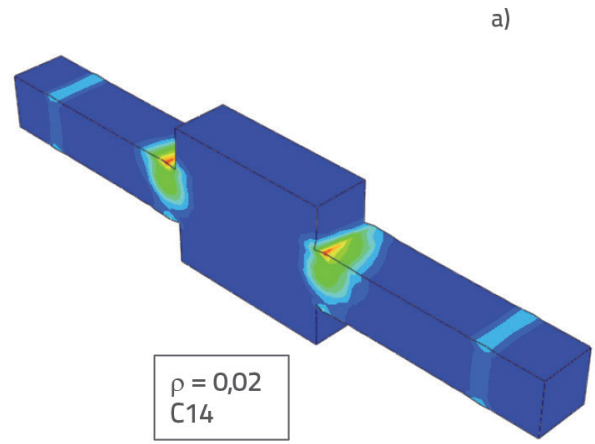

${ }_{2}^{2}$

c)

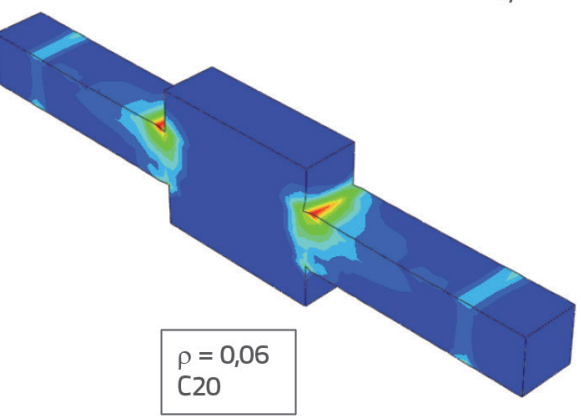

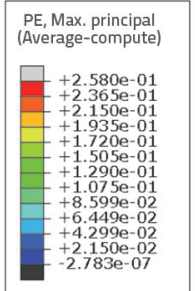

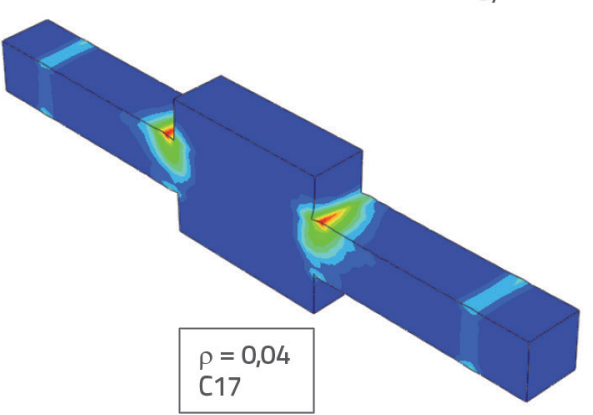

d)

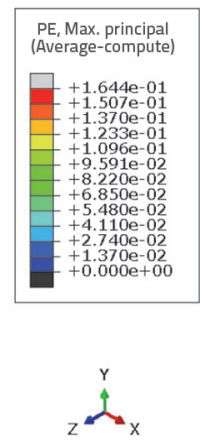

b)

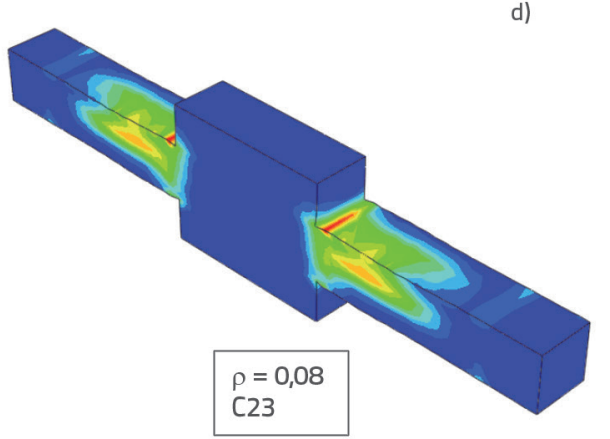

Figure 17. Concrete plastic strain (damage) for specimens with $\emptyset 12 \mathrm{~mm}$ stirrups under biaxial: a) with $\rho=0.02$; b) with $\rho=0.04$; c) with $\rho=0.06$; d) with $\rho=0.08$ ( $\rho$ - longitudinal reinforcement coefficient) 

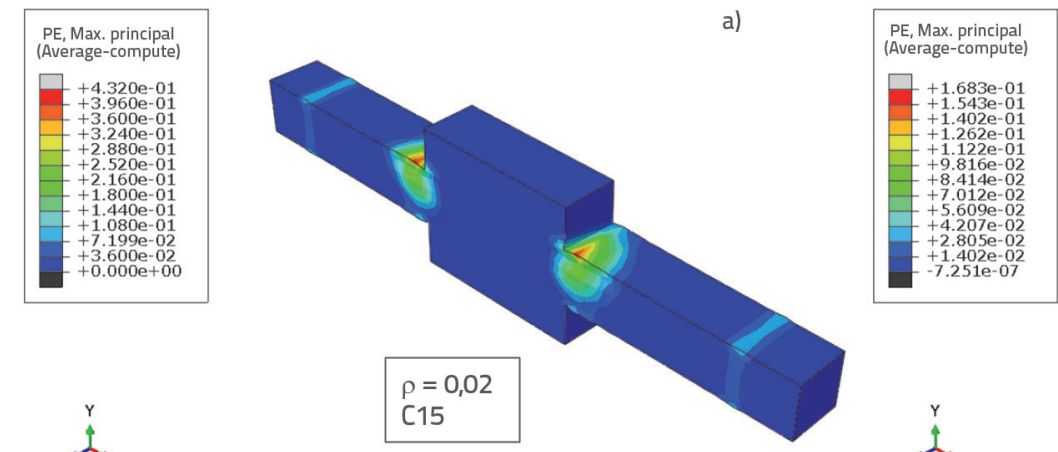

b)

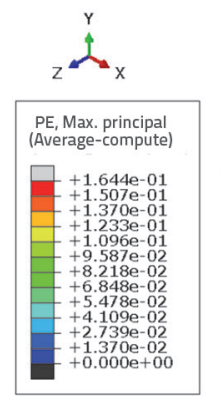<smiles>[Y][Y]([Y])[Y]</smiles>
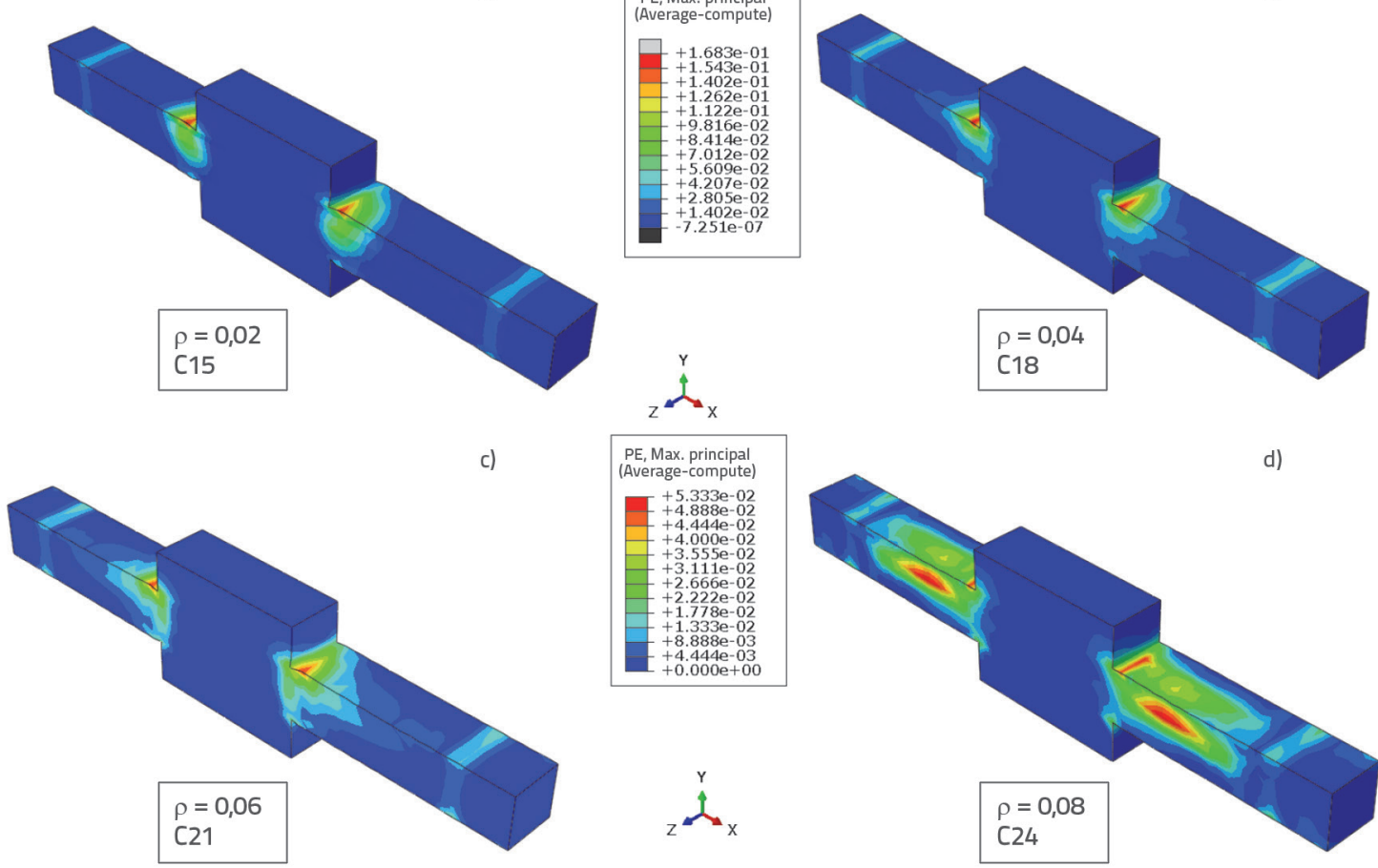

d)

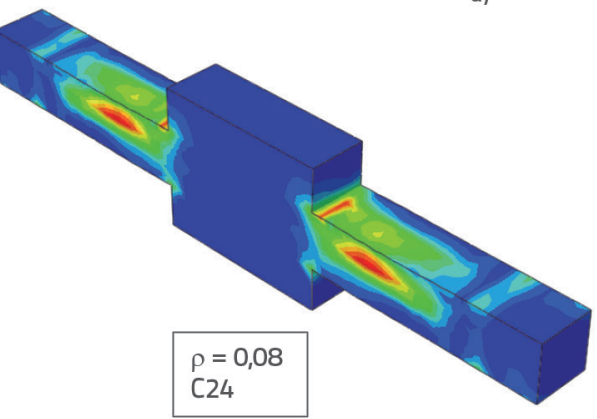

Figure 18. Concrete plastic strain (damage) for specimens with $\emptyset 16 \mathrm{~mm}$ stirrups under biaxial load: a) with $\rho=0.02$; b) with $\rho=0.04$; c) with $\rho=0.06$; d) with $\rho=0.08$ ( $\rho$ - longitudinal reinforcement coefficient)

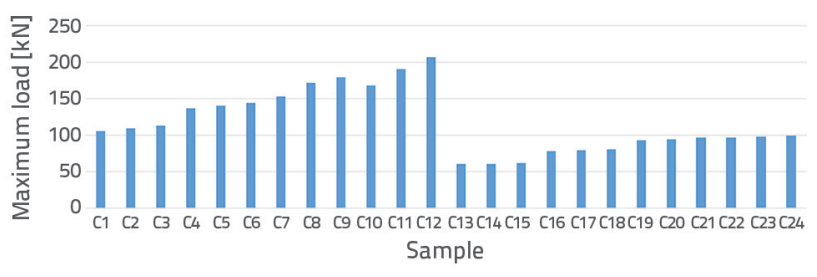

Figure 19. Maximum load of specimens under uniaxial and biaxial load

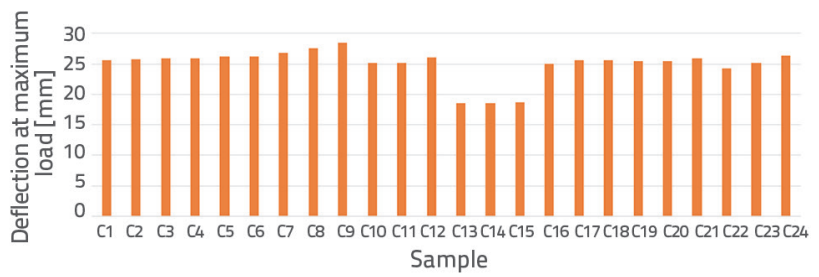

Figure 20. Deflection at maximum load of specimens under uniaxial and biaxial load

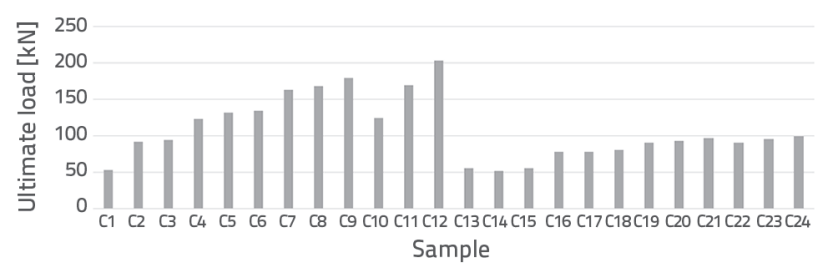

Figure 21. Ultimate load of specimens under uniaxial and biaxial load

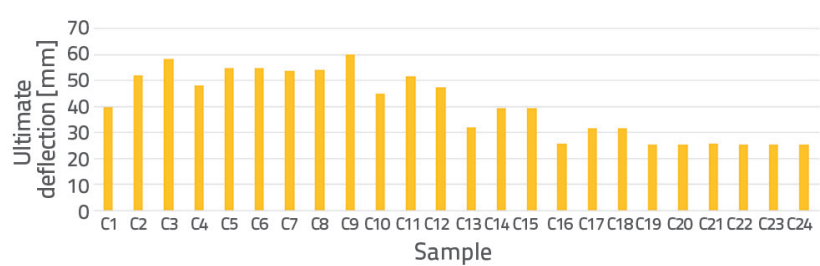

Figure 22. Ultimate deflection of specimens under uniaxial and biaxial load 


\subsection{Effect of longitudinal reinforcement}

The effect of longitudinal reinforcement is based on the numerical FE model results that are presented in figures 7 to 12 and on the data summarized in Table 6 for specimens under uniaxial and biaxial cyclic shear load. It can be observed when the longitudinal reinforcement ratio $\left(\% \rho_{1}\right)$ increases by $0.002,0.004,0.006$ and 0.008 with the same Ash value. The ratio of maximum load for specimens (C4/C1, C7/C1, C10/C1) under uniaxial cyclic shear load increases by $(29$ $\%, 45 \%, 58 \%$ ) and the ratio of ultimate load increases by $(130 \%, 200 \%, 200 \%)$. The ratio of maximum and ultimate loads under biaxial cyclic shear load for specimens (C16/ C13, C19/C 13, C22/C13) increases by (28\%, $53 \%, 60 \%)$ and $(40 \%, 63 \%, 65 \%)$ respectively. Also, similar behaviour can be noted for the remaining specimens with the same Ash value. The significant effect of longitudinal reinforcement ratio $\left(\% \rho_{1}\right)$ increment is clearly shown in Figure 19 and Figure 21. A smaller effect of longitudinal reinforcement ratio $\left(\% \rho_{1}\right)$ increment on the maximum and ultimate deflections is shown in Figure 20 and Figure 22. The number of cycles at maximum and ultimate loads and initial stiffness Ki have a small effect on longitudinal reinforcement increment. In addition, it can be seen from figures 13 to 18 , which show damage to concrete (concrete plastic strain), that the area of tensile cracks reduces when the longitudinal reinforcement ratio $\left(\% \rho_{l}\right)$ increases. Little cracks indicate ductile behaviour of columns specimens.

\subsection{Effect of transverse reinforcement (Ash)}

It is based on numerical results of FE models that are presented in figures 7 to 12 and on the data summarized in Table 6 for specimens under uniaxial and biaxial cyclic shear load. It is also based on comparison results shown in Table 7. The longitudinal reinforcement ratio $\left(\% \rho_{1}\right)$ increases by $0.002,0.004,0.006$ and 0.008 when the Ash value increases by $142.96,254.352$, and $377.17 \mathrm{~mm}$. The following can be observed: maximum load, ultimate load, corresponding deflections, and initial stiffness Ki for uniaxial load increase by $(7.2 \%, 5.5 \%, 16.5 \%, 24 \%),(77 \%, 9.75 \%, 10 \%, 27 \%)$, $(1.1 \%, 1.2 \%, 6.3 \%, 4 \%),(46 \%, 12.5 \%, 13 \%, 4.4 \%)$ and $(0.2$ $\%, 0.2 \%, 0.2 \%, 0.2 \%$, respectively, with each increment of the longitudinal reinforcement ratio. In the case of biaxial load, the increase amounts to $(1.2 \%, 3.9 \%, 5.4 \%, 4 \%),(1.2$ $\%, 4.5 \%, 7.5 \%, 10 \%),(0.5 \%, 2 \%, 1.6 \%, 8.5 \%),(21 \%, 24 \%$, $1 \%, 1 \%)$ and $(0.2 \%, 0.2 \%, 0.2 \%, 0.2 \%)$, respectively. The Ash increment has almost no effect on numbers of cycles at maximum and ultimate loads. This is in accordance with results reported in previous publications [16-19]. Also, figures 13 to 18 shows that an increase in Ash has little effect on the area of tensile cracks.

\subsection{Effect of transverse shear force}

It is based on the results presented in figures 7 to 12 and on the data summarized in Table 6 and also on comparison results shown in Table 8 for specimens with the same longitudinal reinforcement ratio $\left(\% \rho_{1}\right)$ and Ash, when the biaxial cyclic shear load is applied. It can be concluded from differences in behaviour between specimens subjected to uniaxial cyclic shear load (C1 to (12) and specimens subjected to biaxial cyclic shear load (C13 to C24) that the maximum and ultimate loads decrease on an average by $50 \%$ in the case of the biaxial cyclic shear load. In addition, maximum deflection decreased on an average by $10 \%$ while ultimate deflection decreased on an average by $43 \%$. This behaviour is clearly shown in figures 19 to 22 . The numbers of cycles at maximum and ultimate loads decrease significantly due to biaxial cyclic shear load. Also, figures 13 to 18 show the location of maximum tensile cracks area at two opposite corners of the cross-section. The increase of longitudinal reinforcement ratio $\left(\% \rho_{1}\right)$ and Ash reduces the effect of biaxial cyclic shear load significantly.

\section{Conclusions}

This paper presents a numerical investigation on structural behaviour of high strength RC columns subjected to uniaxial and biaxial lateral displacement histories with constant axial load. The effect of longitudinal and lateral reinforcement with uniaxial and biaxial lateral displacement histories is studied. Based on numerical results obtained during this investigation, the following conclusions can be made:

- A good correlation has been established throughout validation by comparison between numerical analysis by ABAQUS and experimental results.

- An increase in longitudinal reinforcement ratio greatly increases maximum and ultimate load of columns, and has less effect on the corresponding deflection, number of cycles and initial stiffness Ki.

- The column load and deformation capacity decreases significantly with biaxial cyclic shear load, compared to uniaxial load. Also, this effect reduces with an increase in longitudinal reinforcement ratio $\left(\% \rho_{l}\right)$ and Ash value.

The above concluding remarks in terms of columns capacity, corresponding deflections, number of cycles, and initial stiffness $\mathrm{Ki}$, suggest that column behaviour is significantly affected by uniaxial load effects combined with biaxial loading. These two aspects cannot be neglected, especially in the case of corner columns.

\section{Acknowledgment}

The Authors would like to extend thanks to Al-Nahrain University, Baghdad, Iraq for their encouragement and support. 


\section{REFERENCES}

[1] Rodrigues, H.: Biaxial seismic behaviour of reinforced concrete columns, Ph.D. thesis, University of Aveiro Civil Engineering Department, 2012.

[2] Bousias, S.N., Verzelleti, G., Fardisand, M.N., Magonette, G.: RC columns in cyclic biaxial bending and axial load, The $10^{\text {th }}$ World Conference on Earthquake Engineering, pp. 3041-3046, Madrid, Spain, 1992.

[3] Saatcioglu, M., Ozcebe, G.: Response of reinforced concrete columns to simulated seismic loading, ACI Structural Journal, 86 (1989), pp. 3-12.

[4] Lejano, B.A.: Investigation of biaxial bending of reinforced concrete columns through fiber method modelling, Journal of Research in Science, Computing and Engineering, 4 (2007), pp. 61-73.

[5] Takizawa, H., Aoyama, M.: Biaxial effects in modelling earthquake response of RC structures, Earthquake Engineering and Structural Dynamics, 4 (1976), pp. 523-552.

[6] Bonet, J.L., Barros, M.H.F.M., Romero, M.L.: Comparative study of analytical and numerical algorithms for designing reinforced concrete sections under biaxial bending, Computers and Structures, 84 (2006) 31-32, pp. 2184-2193.

[7] CEB.: RC frames under earthquake loading, Lausanne Bulletin 220, 1996.

[8] Sfakianakis, M.G., Fardis, M.N.: Bounding surface model for cyclic biaxial bending of RC sections, Journal of Engineering Mechanics, 117 (1991) 12, pp. 2748-2769.

[9] Hussam, K.R., Ibrahim, S.I.H., Abdulkhalik, J.A.: Numerical analysis of RC wall with opening strengthened by CFRP subjected to eccentric loads, GRAĐEVINAR, 69 (2017) 7, pp. 573-580, https:// doi.org/10.14256/JCE.1707.2016

[10] Bousias, S.N., Verzeletti, G., Fardis, M.N., Gutierrez, E.: Loadpath effects in column biaxial bending with axial force, Journal of Engineering Mechanics, pp. 596-605, 1995.

[11] Rodrigues, H., Arêde, A., Varum, H., Costa, A.: Behaviour of RC building columns under cyclic loading: experimental study, Journal of Earthquake and Tsunami, JET, World Scientific Publishing, 2012.

[12] Rodrigues, H., Arêde, A., Varum, H., Costa, A.: Damage evolution in reinforced concrete columns subjected to biaxial loading, Bulletin of earthquake Engineering, 2012.

[13] Low, S. Moehle, J.P.: Experimental study of reinforced concrete columns subject to multiaxial cyclic loading, Earthq. Engrg. Res. Center, Rep. No UCB/EERC 87-14, Univ. of California, Berkeley, 1987.

[14] Bechtoula, H., Kono, S., Watanabe, F.: Experimental and analytical investigations of seismic performance of cantilever reinforced concrete columns under varying transverse and axial loads, Journal of Asian Architecture and Building Engineering, 4 (2005) 2, pp. 467-474.
[15] Nilson, A.H.: Design implications of current research on high strength concrete, High Strength Concrete, SP-87, American Concrete Institute, pp. 85-118, 1985.

[16] Bjerkeli, L., Tomaszewicz, A., Jensen, A.A.: Deformation properties and ductility of high strength concrete, Proc., Second Int. Symposium on High-Strength Concrete, SP-121, American Concrete Institute, pp. 215-238, 1990.

[17] Sakaguchi, N., Yamanobe, K., Kitada, Y., Kawachi, T., Koda, S.: Shear strength of high-strength concrete members, Proc., $2^{\text {nd }}$ Int. Symposium on High-Strength Concrete, SP-121, American Concrete Institute, pp. 155-178, 1990.

[18] Shin, S.W., Kamara, M., Gosh, S.K.: Flexural ductility, strength prediction, and hysteretic behaviour of ultra-high-strength concrete members, Proc., 2nd Int. Symposium on High-Strength Concrete, SP-121, American Concrete Institute, pp. 239-264, 1990.

[19] Sugano, S., Nagashima, T., Kimura, H., Tamura, A., Ichikawa, A.: Experimental studies on seismic behaviour of reinforced concrete members of high strength concrete, Proc., $2^{\text {nd }}$ Int. Symposium on High-Strength Concrete, SP-121, American Concrete Institute, pp. 61-87, 1990.

[20] Hibi, J., Mihara, Y., Otani, S., Aoyama, H.: Behaviour of reinforced concrete columns using high strength concrete after flexural yielding, Trans. Japan Concr. Inst., 13 (1991), pp. 395-402.

[21] Matamoros, A., Sozen, M.: Drift Limits of High-Strength Concrete Columns Subjected to Load Reversals." Journal of Structural Engineering-ASCE, 129 (2003) 3, pp.297.

[22] ABAQUS.: Standard version 6.13-4 and ABAQUS standard users' manual, Hibbitt Karlsson and Sorensen Inc, 2014.

[23] FEMA.: Improvement of Nonlinear Static Seismic Analysis Procedures, Washington, DC, 2005.

[24] Carreira, D.J., Chu, K.H.: Stress-strain relationship for plain concrete in compression, ACI Struct. J., 82 (1985) 11, pp. 797-804.

[25] Hordijk, D.: Local approach to fatigue of concrete, Ph.D. thesis, Delft University of Technology, Delft, The Netherland, 1991.

[26] Lee, P.S., Noh, H.C.: Inelastic buckling behaviour of steel members under reversed cyclic loading, Engineering Structures, 32 (2010), pp. 2579-2595.

[27] White, C.S., Bronkhorst, C.A., Anand, L.: An improved isotropickinematic hardening model for moderate deformation metal plasticity, Mechanics of Materials, 10 (1990), pp. 127-147.

[28] Kojic, M., Bathe, K.J.: Inelastic analysis of solids and structures, Springer, New York, 2005.

[29] Ucak, A., Tsopelas, P.: Constitutive model for cyclic response of structural steels with yield plateau, Journal of Structural Engineering, 137 (2011), pp. 195-206. 